NBER WORKING PAPER SERIES

\title{
CONTRACTING IN PEER NETWORKS
}

\author{
Peter M. DeMarzo \\ Ron Kaniel \\ Working Paper 28378 \\ http://www.nber.org/papers/w28378 \\ NATIONAL BUREAU OF ECONOMIC RESEARCH \\ 1050 Massachusetts Avenue \\ Cambridge, MA 02138 \\ January 2021
}

The research leading to these results has received funding from the European Research Council under the European Union's Seventh Framework Programme (FP7/2007-2013) / ERC Grant Agreement no.[312842]. We are grateful to Shai Bernstein, Gian Luca Clementi, Florian Ederer, Willie Fuchs, Denis Gromb, Adriano Rampini, Jeff Zwiebel, and seminar and conference participants at Amherst, Baruch College, Boston University, Cheng Kong Graduate School of Business, Chinese University of Hong Kong, Drexel University, Emory, Federal Reserve Bank of New York, Frankfurt School of Finance and Management, Fudan, IDC Herzeliya, INSEAD, John Hopkins, London School of Economics, National University of Singapore, Pompeu Fabra and Barcelona GSE, Rutgers, Rice, Singapore Management University, Tel Aviv University, Tulane, University of Chicago, University of Gothenburg, University of Hong Kong, University of Houston, University of Lugano, University of Melbourne, University of Minnesota, University of Rome, University of Texas at Dallas, University of Toronto, Vienna University of Economics and Business, Washington University in St. Louis, Annual Meeting of the Western Finance Association, Utah Winter Finance Conference, Finance Theory Group conference, Luxembourg Asset Management Summit, and the Annual Meeting of the American Finance Association for helpful comments. An earlier version of this paper was circulated under the title "Relative Pay for Non-Relative Performance: Keeping up with the Joneses with Optimal Contracts." The views expressed herein are those of the authors and do not necessarily reflect the views of the National Bureau of Economic Research.

NBER working papers are circulated for discussion and comment purposes. They have not been peer-reviewed or been subject to the review by the NBER Board of Directors that accompanies official NBER publications.

(C) 2021 by Peter M. DeMarzo and Ron Kaniel. All rights reserved. Short sections of text, not to exceed two paragraphs, may be quoted without explicit permission provided that full credit, including $\odot$ notice, is given to the source. 
Contracting in Peer Networks

Peter M. DeMarzo and Ron Kaniel

NBER Working Paper No. 28378

January 2021

JEL No. D85,D86,G3,G4,J3

\begin{abstract}
$\underline{\text { ABSTRACT }}$
We consider multi-agent multi-firm contracting when agents benchmark their wages to a weighted average of their peers, where weights may vary within and across firms. Despite common shocks, compensation benchmarking can undo performance benchmarking, so that wages load positively rather than negatively on peer output. Although contracts appear inefficient, when a single principal commits to a public contract, the optimal contract hedges agents' relative wage risk without sacrificing efficiency. Moreover, the principal can exploit any asymmetries in peer effects to enhance profits. With multiple principals, or a principal that is unable to commit, a "rat race" emerges in which agents are more productive, but wages increase even more, reducing profits and undermining efficiency. Effort levels are too high rather than too low, and can exceed first best. Wage transparency and disclosure requirements exacerbate these effects.
\end{abstract}

Peter M. DeMarzo

Graduate School of Business

Stanford University

Stanford, CA 94305

and NBER

pdemarzo@stanford.edu

Ron Kaniel

University of Rochester

Simon School of Business

500 Wilson BLVD

Carol Simon Hall

Box 270100 (Room CS3-312)

ron.kaniel@simon.rochester.edu 


\section{Introduction}

Contract and incentive theory has provided powerful insight into the optimal design of compensation contracts. Chief among them, for instance, is the idea that contracts should provide higher compensation when output suggests that the agent was more likely to have engaged in desired behavior. In particular, Holmstrom's (1992) Informativeness Principle states that any measure of performance that reveals information about the agent's effort should be included in the compensation contract. A prime example is the use of Relative Performance Evaluation (RPE), in which the agent's performance is measured relative to an average of her peers in order to filter out common sources of noise. In other words, optimal contracts should not "pay for luck" due to aggregate shocks, but only pay for indicators of individual performance. Yet despite this clear benefit, such performance benchmarking is observed much less frequently than theory would predict. ${ }^{1}$

But while principals may care about relative performance, agents may also care about their relative wage. In this paper, we consider such a setting and explore its consequences. Specifically, we suppose agents have a "keeping up with the Joneses" component to their preferences, and compare their wage to a weighted average of the wages of their peers. We allow for a general network of peer relationships, where the strength of comparison may vary both within and across firms based on proximity, salience, or other factors. We fully characterize optimal contracts in this setting, and show that many standard contracting results may be overturned - contracts may reward agents for peer performance, and, despite seemingly weaker incentives, equilibrium effort may exceed the first best.

In particular, we show that when peer effects are strong enough, compensation benchmarking undoes performance benchmarking, leading to wage contracts that positively load on peer (or team) output. But although these contracts appear inefficient, we show that this effect on its own does not diminish incentives nor welfare. In fact, despite what appear to be "lower power" incentives, when there are multiple principals, or if contracts are privately negotiated, a

\footnotetext{
${ }^{1}$ For empirical evidence of "pay for luck" in the context of CEO compensation, see e.g. Murphy (1985), Coughlan and Schmidt (1985), Antle and Smith (1986), Gibbons and Murphy (1990), Janakiraman, Lambert, and Larker (1992), Garen (1994), Aggarwal and Samwick (1999a,b), Murphy (1999), Frydman and Jenter (2010), and Jenter and Kanaan (2014).
} 
"rat race" ensues that leads to higher wages and effort that may even exceed the first best. And although rat race effects increase productivity, wages rise even more, causing a decline in profits. Measures that increase external wage transparency (such as public disclosure requirements) are likely to exacerbate these rat race effects. Finally, if peer-effects are asymmetric, principals can exploit the asymmetry by reallocating effort to less visible agents. Though production is inefficient, incentive costs fall and, somewhat surprisingly, profits and welfare rise relative to when agents' preferences lack peer-effects.

Our model includes many agents who take hidden effort to produce output that is subject to both common and idiosyncratic shocks. Agents receive a compensation contract which specifies their wage as a function of their own output as well as the output of others. Agents are risk averse and have preferences that are increasing in both their own wage as well as the difference between their own wage and a weighted-average benchmark of their peers' wages. We allow these weights to depend on a general peer-network structure so that, for example, agents may put more weight on close colleagues versus peers elsewhere in the firm (or in other firms). We then consider the sensitivity of the optimal contract to both the strength of agents' relative wage concerns, as well as the peer-network structure, and evaluate implications for welfare.

We begin in Section 2 by analyzing the RPE benchmark in a multi-agent model absent any peer effects, and show that as expected, compensation in the optimal contract is based on a measure of the agent's relative performance; that is, compensation is positively related to the agent's own output and negatively related to the output of others, in relative proportions that depend on the correlation between agents' output. A key prediction of RPE is therefore that the incentive component of wages should be negatively correlated. Additional standard predictions are that equilibrium effort is reduced relative to the first best (in order to limit agents' risk) and that the optimal contract for each agent is independent of the contracts of others (and therefore, independent of how they are determined, whether they are disclosed or not, etc.).

In Section 3, we consider optimal contracts with peer network effects. Because agents care about their compensation relative to their peers, the incentives for each agent will depend on both their own contract and the contracts offered to all other agents. Principals independently choose contracts for agents on their teams, which are composed of one or many agents. We assume (for 
now) that agents see the full set of contracts offered by their principal to their team before deciding to accept or reject their own contract.

As an initial baseline, we first consider a setting in which peer weights are symmetric (determined, for example, by a distance metric) and there is a single principal. We then demonstrate an important welfare equivalence result: the optimal contracts for a single principal will fully hedge peer effects and lead to the same productivity, profitability, and welfare as the RPE benchmark. On the other hand, observed contracts will have markedly different sensitivities than those predicted by RPE. In particular, to limit relative wage risk, contracts may positively load on peer output (contradicting RPE). This result therefore implies that empirically observed deviations from RPE need not be inefficient.

Next, we consider the case with multiple principles independently setting contracts for distinct teams. In this case, there is an important "rat race" externality across teams, as principals do not account for the impact of raising the wage for their team on the welfare of agents on other teams. As a result, in equilibrium average wages and productivity are higher, but profitability and welfare are lower, than in the RPE or single principal benchmark. We show that the rat race inefficiency increases with the number of principals, the strength of peer effects, and the importance of external (non-teammate) peers. In fact, the rat race effect can be so extreme that equilibrium effort exceeds the first best, in stark contrast to standard agency models.

We then explore in greater detail the consequences of peer effects for wage correlations. We show that wage correlations are the same in both the single and multi-principal case and are therefore solely related to the peer effects and independent of welfare. We show that wage sensitivity to peer-performance increases monotonically with the strength of peer-effects, leading to positive wage correlation, consistent with empirical evidence on wage compression within firms. ${ }^{2}$ We characterize specific cases, and show that wage contracts load more positively on "nearby" versus more distant peers. Thus, for example, if agents care more about peers within

\footnotetext{
${ }^{2}$ For example, Silva (2016) and Gartenburg and Wulf (2016) document wage convergence in multidivisional firms, which is heightened by geographic or social proximity. Shue (2013) shows similar wage convergence across executives who were former classmates. While inequity aversion is a possible explanation for wage compression (see Englmaier and Wambach (2010) and Koszegi (2014)), our analysis demonstrates that peer effects are sufficient.
} 
their own firm or team, we find that optimal wage contracts will always have a positive exposure to the relative performance of their team. ${ }^{3}$

In Section 4 we allow for peer relationships to be asymmetric. In that case, some agents may be "more visible" than others in the sense that their wage is more salient to their peers. In that case, the principal can exploit this asymmetry and use the departure from RPE to increase profits. Specifically, it is optimal to reallocate effort, and therefore compensation, to less visible agents. Although this reallocation is technologically inefficient, it lowers the principal's expected cost of providing incentives and thereby overall output and profits.

In Section 5 we consider the consequences of alternative disclosure environments. Importantly, in the RPE framework wage and contract disclosure has no effect. Here, on the other hand, disclosure interacts with peer effects. For example, we show that if the principal can privately negotiate individual wage contracts, the ability to do so exacerbates the rat race effect, raising equilibrium wages and lowering profits. Indeed, a single principal that cannot commit not to renegotiate may have worse outcomes than would occur if all contracts were negotiated by independent principals.

We also consider the effect of greater wage transparency across teams or firms. Wage transparency is likely to make peer comparisons more salient, and thereby exacerbate the rat race externalities across teams; as a result, we expect average wages to rise and their correlation to increase. We also show that when incentive contracts are disclosed ex ante, there is an additional incentive to increase the contract loading on external peers, and equilibrium effort is distorted downward (and can be below second best).

\subsection{Related Literature}

The key premise of our paper is that some agents have Keeping up with the Joneses (KUJ)type preferences in which they care about their relative wage with respect to their peers. Our objective is to understand implications for contracting of such preferences. Evidence supporting the presence of KUJ / relative wage-based preferences has accumulated from multiple disciplines

\footnotetext{
${ }^{3}$ Ibert, Kaniel, Van Niuwerburgh, and Vestman (2018) find that fund managers elasticity of pay to fund family revenue, excluding revenue managed by the given manager, is comparable to that of manager revenue.
} 
and research designs. Early evidence was derived mostly from survey questions regarding happiness and satisfaction (for example: Luttmer 2005, Ferrer-i-Carbonell 2005). ${ }^{4}$ More recently, researchers have utilized interesting natural experiments as well as FMRI evidence to further bolster support for the presence of such preferences. Kuhn et.al. (2011) utilize the Dutch Postcode Lottery as a natural-experimental. They show that while the majority of BMW winners do not own a BMW six months after the lottery, having an immediate neighbor win the lottery raises the probability that a household will buy a car in the next six months by close to 7 percentage points. Card et.al. (2012) provide field-based confirmation of relative pay comparisons by randomized manipulation of revelation of information on coworkers' salaries. ${ }^{5}$ Fliessbach et.al. (2007), using two adjacent FMRI scanners, provide neuro-physiological foundations of relative comparison by showing that variation in a subject's relative payment affects blood oxygenation level-dependent responses in the ventral striatum, a brain region known to be involved in processing of rewards. In a similar setting, Dohem et. al. (2011) find effects of own and others' income on activation in the ventral striatum are equal and opposite.

In providing evidence for relative wage preferences the literature has considered a variety of different peer groups: co-workers (Clark and Oswald (1996)); neighbors (Luttmer (2005), and Kuhn et.al. (2011)); household members (Clark (1996)); siblings (Neumark and Postlewaite (1998)); caste members in India (Carlsson, Gupta, and Johansson-Stenman (2009)); past MBA classmates (Shu (2013)); division managers within the same firm (Duchin, Goldberg, and Sosyura (2014)).

More recently, Bouwman (2013) finds that CEO pay is strongly correlated with that of geographically-close CEOs. After carefully controlling for a host of potentially confounding effects, she concludes that KUJ preferences is the most plausible explanation. ${ }^{6}$ Two result in

\footnotetext{
${ }^{4}$ See Heffetz and Frank (2011) for a review.

${ }^{5}$ Following a court decision on California's "right to know" law, the Sacramento Bee newspaper established a website that made it possible to search for the salary of any state employee. After the sites launch, the researchers contacted a random subset of University of California employees informing them about it, and a few days later surveyed all campus employees about job satisfaction.

${ }^{6}$ Bouwman (2013) rules out three key competing hypothesis. First, that geography may introduce commonalities in the performance-relevant characteristics of CEOs that firms in a given area emphasize in their selection of CEOs. Second, that firms may follow "leading" firms in the vicinity in setting CEO pay. Third, local labor market competition for CEOs.
} 
Bouwman (2013) are especially pertinent to our conceptual peer group framework. First, correlations are higher for locally connected CEOs, where connectedness is inferred from rolodex connections. Second, and more striking, CEO pay is related to the compensation of high flying sports stars in their areas, highlighting that peers need not be in the same profession. Shue (2013) demonstrates wage convergence across executives who were former classmates, and shows the effect is stronger for section mates. Our modeling framework contributes to the literature by allowing for a flexible structure of peer preferences, with differential peer preference sensitivities for a given agent and across agents. These heterogeneous peer preference sensitivities implicitly aggregate a wide variety of factors differentially impacting the sensitivity to different agents: the size and complexity of firms they work for or manage, common background, geographical proximity, social interactions, person specific psychological traits etc.

While Keeping/Catching up with the Joneses and habit formation preferences have been used in asset pricing applications starting with Abel (1990), they have received much less attention in explaining behavior in the corporate finance domain. Ederer and Patacconi (2010) introduce status considerations into a tournament setting analyzing implications for the provision of incentives. Goel and Thakor (2010) use envy-based preferences for managers to explain merger waves. ${ }^{7}$ Dur and Glazer (2008) consider the optimal contract, with contractible effort, for an employee that is envious of his employer. Goel and Thakor (2005) consider within firm capital allocation decisions of division managers where each manager derives direct utility from wages, and in addition envies both the wages of other managers and their capital allocation as well. Their analysis focuses on induced capital distortions, ignoring the moral hazard and contracting considerations which are the focus of our analysis.

The central theme of our analysis is to underscore contracting implications of KUJ preferences. We contribute to the literature by showing that these preferences reverse central results from standard contract theory: Compensation can load positively rather than negatively on outputs of other agents; effort levels are too high rather than too low and can even exceed first

\footnotetext{
${ }^{7}$ While closely related, envy refers to a disutility from having a lower wage than one's peer, whereas keeping up with the Joneses preferences also include a benefit from having a higher wage.
} 
best $;^{8}$ disclosure of contracts may reduce efficiency, instead of being neutral or better; and when peer effects are asymmetric, the principal's profits can be higher than when agents have standard preferences that do not depend on peers.

There is extensive empirical literature that has for the most part rejected the RPE hypothesis that $\mathrm{CEO}$ compensation should depend on relative performance, and so be negatively related to the performance of peers. Most of the evidence documents a positive relation, in direct contrast to the standard RPE prediction. ${ }^{9}$ A few have noted that KUJ preferences can provide a resolution for these findings within the agency model, as these preferences lead to optimal contracts with a reduced magnitude of RPE and, when strong enough, even a positive dependence of pay on external performance measures (Fershtman, Hvide, and Weiss (2003), Itho (2004), Miglietta (2008), Bartling (2011), Liu and Sun (2016)). Bartling and von Siemens (2010) consider the impact of envy on contracts in a general moral hazard model when a principal hires two agents that are envious of each other. They argue that with risk-averse agents and without limited liability envy can only increase the costs of providing incentives. The scope of their analysis is limited by the fact that they do not derive explicit optimal contracts. We, on the other hand, show that under the optimal contract a principle that hires multiple agents can in fact attain at least the second best, implying no loss in efficiency. In fact, when the collective peer weight put on agents by others is heterogeneous, the principal's profits are higher than when agents have standard preferences, implying increased efficiency. More generally, none of the above papers scale agents' outside options to make appropriate welfare comparisons as preferences vary. In contrast, our analysis provides general conditions for efficiency as well as meaningful comparative statics with regard to the strength of relative wage concerns.

In addition to providing a more comprehensive welfare comparison, our analysis considers a market-wide equilibrium with multiple principals, employing potentially multiple agents, within a general network structure of potential peer relationships. This setting allows us to additionally analyze contracting externalities across principals. Finally, we investigate the impact of the

\footnotetext{
${ }^{8}$ The predicted link between peer effects and effort is consistent with evidence in Ghazala, A. and N. Iriberri (2010), which utilizes a natural experiment to show that adding to a students' report cards their average grade over all subjects, as well as the class average over all subjects and students led to an increase of 5\% in students' grades.

${ }^{9}$ Examples include: Antle and Smith (1986), Barro and Barro (1990), Jensen and Murphy (1990), Janakiraman, Lambert and Lacker (1992), Hall and Liebman (1998), Joh (1999), Aggarwal and Samwick (1999a,b), Bertrand and Mullainathan (2001), and Garvey and Milbourn (2003).
} 
disclosure environment, both inside the firm and across firms, on contracts and efficiency. For example, we predict that compensation disclosure mandates will increase rat race incentives and raise wages, consistent with the findings in Park, Nelson, and Huson (2001), Perry, and Zenner (2001), Schmidt (2012), Gipper (2016) and Mas (2016).

\section{A Model of Peer-Contracting}

\subsection{Peers and Preferences}

We consider a setting with a set $\mathcal{N}$ of $N=|\mathcal{N}| \geq 2$ identical agents. We make the standard assumption that the utility of each agent $i$ is increasing in his own wage, $w_{i}$, and decreasing in his hidden choice of effort, $a_{i}$. We depart from the usual principal-agent framework, however, by assuming that agents also care about their wage relative to that of their peers. We model this preference by assuming agent $i$ benchmarks his own wage against a weighted average of the wages of his peers, ${ }^{10}$

$$
w_{-i} \equiv \sum_{j \in \mathcal{N}} \mu_{i j} w_{j} \text { with } \mu_{i j}=\mu_{j i} \geq 0, \mu_{i i}<1, \text { and } \sum_{j \in \mathcal{N}} \mu_{i j}=1
$$

The weights $\mu_{i j}$ capture peer network effects in which agents may care more about the wages of some peers versus others. For now, we restrict the weights to be symmetric as we interpret them to be a measure of "closeness"; for example, the wages of peers in the same office / firm / industry may be more salient and thus more important. For simplicity, we assume the set of peers is irreducible (i.e. for any pair $(i, j)$ there is a sequence connecting them with positive weights).

We assume agent $i$ derives utility from his "relative wage" $v_{i}$, which is increasing in his own wage and decreasing in the peer benchmark,

$$
v_{i} \equiv w_{i}+\left(\frac{\delta}{1-\delta}\right)\left(w_{i}-w_{-i}\right)=\left(\frac{1}{1-\delta}\right) w_{i}-\left(\frac{\delta}{1-\delta}\right) w_{-i} \text { with } \delta \in[0,1)
$$

\footnotetext{
${ }^{10}$ It is natural to assume $\mu_{i i}=0$ so that the benchmark includes only the wages of others. We allow $\mu_{i i}>0$ for additional generality (see footnote 11 ) but restrict $\mu_{i i}<1$ to ensure some weight is put on peers.
} 
Here $\delta$ captures the strength of agents' relative wage concerns, with $\delta=0$ corresponding to the standard model without peer effects $\left(v_{i}=w_{i}\right) .{ }^{11}$ Finally, to avoid wealth effects and for tractability, we assume agents have CARA utility $u\left(c_{i}\right)=-\exp \left(-\lambda c_{i}\right)$, where $c_{i}$ is the "adjusted consumption" level

$$
c_{i} \equiv v_{i}-\psi\left(a_{i}\right)
$$

and $\psi$ measures the cost of effort, which we assume for simplicity is quadratic: $\psi(a)=\frac{1}{2} k a^{2}$.

This specification of the agent's utility incorporates several properties that are both natural and important given our goal of comparing welfare and contract design for different levels of relative wage concerns $\delta$. First, $v_{i}$ is linear in wages so that changing $\delta$ has no direct effect on the agent's risk aversion (and therefore the magnitude of the agency friction). Second, the weights in $v_{i}$ sum to one, so that peer effects do not alter the aggregate tradeoff between income and effort. Of course, because wage variation is necessary for incentive provision, agents with peer-dependent preferences experience additional disutility associated with the risk of their wage differentials, which will have important effects on optimal contracts.

\subsection{Production and Wages}

The production technology has additive shocks, so that the output $q_{i}$ of agent $i$ is equal to a known constant $q_{0}>0$ plus effort plus noise: ${ }^{12}$

$$
q_{i} \equiv q_{0}+a_{i}+\epsilon_{i}
$$

\footnotetext{
${ }^{11}$ Many of our results also apply if $\delta<0$ so that agents are altruistic, but we do not analyze that case here. Note also that $\mu_{i i}>0$ is equivalent to reducing the weight agent $i$ places on others to $\hat{\delta}_{i}=\delta\left(1-\mu_{i i}\right) /\left(1-\delta \mu_{i i}\right)$. Thus, by adjusting $\mu_{i i}$ for each $i$ we can consider a setting in which agents differ in terms of the strength of their concern for peers.

${ }^{12}$ We can think of $q_{0}$ as corresponding to some component of output that is not subject to moral hazard. We assume it is large enough such that hiring an agent is strictly profitable.
} 
The random shocks $\epsilon_{i}$ are joint normal with mean zero and variance $\sigma_{\epsilon}^{2}$, and have a pairwise correlation of $\rho \in[0,1) .^{13}$

Given the production technology and preferences, the first-best effort level maximizes $a_{i}-\psi\left(a_{i}\right)$, which is solved by $a^{f b} \equiv k^{-1}$. Effort choices are hidden, however, and therefore appropriate compensation contracts are needed to motivate the agent. Even absent relative wage concerns, the correlation between output shocks implies that optimal contracts will depend on both own output and peer output. We restrict attention to linear compensation contracts $y_{i}$ of the form: ${ }^{14}$

$$
w_{i}=y_{i 0} q_{0}+y_{i i} q_{i}+\sum_{j \in \mathcal{N}_{i}} y_{i j} q_{j}=y_{i} \cdot q
$$

where $\mathcal{N}_{i}=\mathcal{N} \backslash\{i\}$ is the set of $i$ 's peers, $y_{i 0}$ determines the constant component of the wage, $y_{i i}$ is the sensitivity of the wage to $i$ 's own output, and $y_{i j}$ is the sensitivity of $i$ 's wage to the output of peer $j .{ }^{15}$ Of particular interest is the contract's "relative sensitivity" to peer output, which we define by:

$$
\phi_{i} \equiv \sum_{j \in \mathcal{N}_{i}} \frac{y_{i j}}{y_{i i}}
$$

To evaluate payoffs, note that with normally distributed shocks, linear contracts, and CARA utility, agents will have mean-variance preferences. Therefore, given normally distributed consumption $c$, we can evaluate the agent's utility in terms of the corresponding certainty equivalent consumption level

$$
u^{-1}(E[u(c)])=E[c]-\frac{1}{2} \lambda \operatorname{Var}(c)
$$

\footnotetext{
${ }^{13}$ Equivalently, we can write the shocks as $\epsilon_{i}=\sigma_{\epsilon}\left[\sqrt{1-\rho} \eta_{i}+\sqrt{\rho} \eta_{c}\right]$, with $\eta$ independent standard normal. We assume for simplicity that the pairwise correlations are equal so that the relative performance evaluation benchmark is uniform across all workers and all contract asymmetries are due solely to peer effects in preferences.

${ }^{14}$ Goukasian and Wan (2010) demonstrate optimality of linear contracts with one principal and two agents in a continuous time context as in Holmstrom and Milgrom (1987). Similar results may be possible in our more general framework, but we have not pursued that here.

${ }^{15}$ It is not essential that $q_{0}$ in (5) is the same as the constant component in (4), but because the constant in (5) is arbitrary (as long as it is nonzero), we use the same term for notational convenience.
} 
We assume that each agent has an outside option with certainty equivalent $c_{0} \leq q_{0}$, which implies that hiring an agent is always efficient (even without providing incentives).

\subsection{Teams}

The contract for each agent is set by a risk neutral principal. Each principal manages a distinct set of agents, which we refer to as a team. Teams may correspond to different firms, or different divisions or groups within a firm. The set of teams is a partition of the set $\mathcal{N}$ of agents. The objective of the principal for team $I \subseteq \mathcal{N}$ is to offer a set of contracts to maximize the team's expected output net of wages paid:

$$
\pi_{I} \equiv E\left[\sum_{i \in I}\left(q_{i}-w_{i}\right)\right]
$$

The principal must respect each agent's incentive compatibility constraint, which given (7) can be written as

$$
a_{i} \in \arg \max E\left[c_{i}\right],
$$

and the participation constraint,

$$
E\left[c_{i}\right]-\frac{1}{2} \lambda \operatorname{Var}\left(c_{i}\right) \geq c_{0}
$$

The maximum team size is the total population; in this case one principal manages all peers (e.g. workers within a single firm). At the other extreme, each agent may have his own principal (for example, CEOs with distinct boards). In general, an agent will have peers both within and outside his team. We refer to agents on the same team as teammates or "internal" peers, whereas agents on different teams are "external" peers. We denote the total number of peers and the number of people on a team by

$$
n \equiv\left|\mathcal{N}_{i}\right|=N-1 \text { and } N_{I} \equiv|I| \in[1, N]
$$

\subsection{Contracting}

We assume each principal chooses contracts for their team taking as given the contracts offered and actions taken by all other teams. That is, the contracts proposed by the principal for team $I$ are visible to all members of the team, but not to principals or agents outside the team. (We 
will consider other disclosure assumptions in Section 5.) The timing of the contracting problem faced by team $I$ is shown in Figure $1 .{ }^{16}$

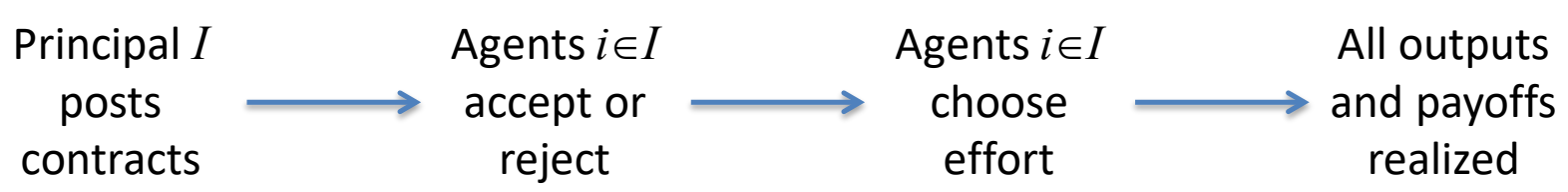

Figure 1: The Contracting Game for a Single Principal

The principal-agent problem for team $I$ (taking all other contracts and actions as given).

Figure 2 depicts the different channels that may affect the agent's utility. The solid blue lines correspond to the standard principal-agent problem in which agents' output is uncorrelated. In that setting, the optimal wage contract depends only on the agent's own output. When there are multiple agents with correlated output shocks a new information channel is introduced, depicted by the dashed green lines. In this case, the principal can use the information in the output of others to filter out any common component of the agent's productivity shock, and relative performance evaluation (RPE) becomes optimal.

Finally, the dotted orange lines represent the new channels introduced when peer effects cause agents to also care about their relative wage. Each agent is now exposed to both the risk and the level of their wage differential with their peers. This interaction introduces two new aspects to the optimal contract. First, the principal may adjust the contract to hedge the agent against peer wage risk. Second, the principal may distort the agent's effort in order to affect the level of the average peer wage.

\footnotetext{
${ }^{16}$ Note that this timing presumes that contract details are visible within a team, but only ex-post output and wages are visible across teams. Wages and output are likely to be more easily observed even externally due to standard reporting requirements as well as consumption and investment effects. That said, we explore the consequences of alternative information structures in Section 5.
} 


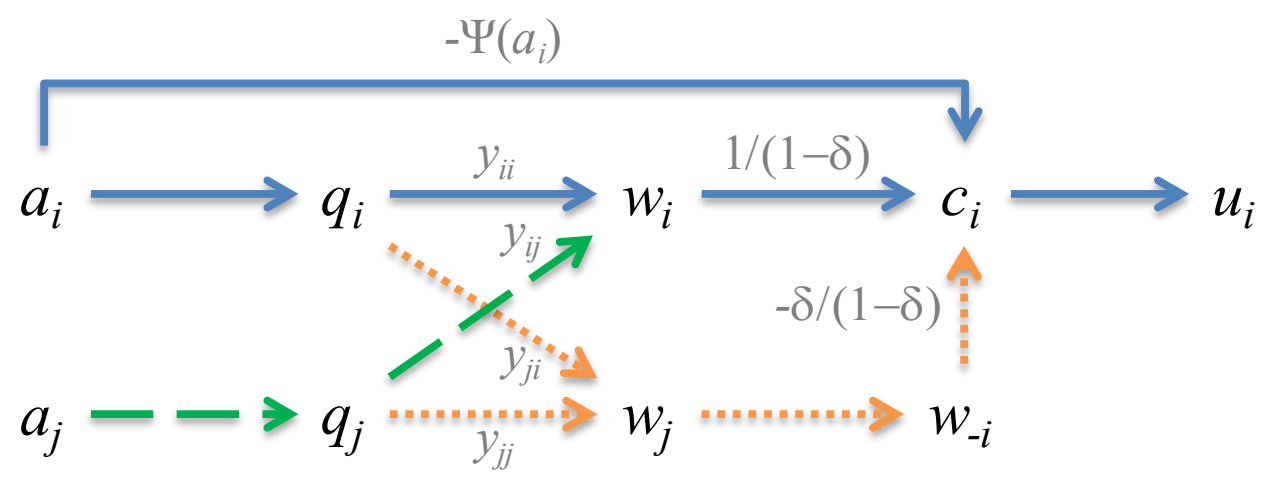

Figure 2: Alternative Channels by which Effort and Output Impact Utility

Standard principal-agent model is shown in solid blue. The information channel for relative performance evaluation is shown in dashed green. The dotted orange channel represents relative wage concerns.

\subsection{The RPE Benchmark}

As an important benchmark, before we consider peer effects we first solve for the optimal contract in the benchmark case without peer effects and obtain the standard prediction that optimal contracts should utilize relative performance evaluation (RPE): each agent's wage should have a negative sensitivity to the output of his peers.

Consider the standard setting with $\delta=0$ so that peer effects are absent. In that case, the contracting problem for agent $i$ does not depend on the wage contracts of other agents. The only interdependence between agents arises from the positive correlation between agents' shocks. As a result, it is optimal for the principal to reduce the agent's risk by "filtering out" the common component of output when assessing the agent's performance.

In particular, the contract can reward the agent for his performance relative to a benchmark of output based on the performance of his peers. The efficient benchmark will minimize the agent's exposure to the common risk factor. This benchmark is the multiple $\bar{\theta}$ of the average peer performance $q_{-i}=\frac{1}{n} \sum_{j \in \mathcal{N}_{i}} q_{j}$ that minimizes the residual variance:

$$
\bar{\theta} \equiv \underset{\theta}{\arg \min } \operatorname{Var}\left(q_{i}-\theta q_{-i}\right)=\frac{\operatorname{Cov}\left(q_{i}, q_{-i}\right)}{\operatorname{Var}\left(q_{-i}\right)}=\frac{\rho}{\rho+\frac{1}{n}(1-\rho)} \in[0,1) .
$$

Given this benchmark, the residual uncertainty is given by 


$$
\bar{\sigma}^{2} \equiv \operatorname{Var}\left(q_{i}-\bar{\theta} q_{-i}\right)=(1-\rho \bar{\theta}) \sigma_{\epsilon}^{2}>0
$$

The volatility $\bar{\sigma}$ of the optimal signal available to the principal determines the magnitude of the agency problem, as it is costly for the agent to bear this risk. Together the parameters $(\bar{\theta}, \bar{\sigma})$ summarize the information externalities in our model.

Lemma 1 (Relative Performance Evaluation). Absent relative wage concerns $(\delta=0)$, the optimal contract for each agent solves

$$
\max _{y_{i .}} a_{i}-\Psi\left(a_{i}\right)-\frac{1}{2} \lambda \operatorname{Var}\left(c_{i}\right) \quad \text { s.t. } a_{i}=y_{i i} / k
$$

Optimal contracts and actions are given by

$$
y_{i i}^{*}=\frac{1}{1+k \lambda \bar{\sigma}^{2}}, y_{i j}^{*}=-\frac{1}{n} \bar{\theta} y_{i i}^{*}, \text { and } a_{i}^{*}=y_{i i}^{*} / k
$$

The constant term $y_{i 0}^{*}$ is set so that the expected wage is $E\left[w_{i}\right]=c_{0}+\frac{1}{2} a_{i}^{*}$, and the expected profit per agent is $\pi_{i}^{*}=q_{0}-c_{0}+\frac{1}{2} a_{i}^{*}$.

Proof: See Appendix.

The intuition for this result is as follows. Equation (14) states that the optimal contract maximizes the expected output net of the cost of effort and risk-bearing. The wage parameter $y_{i i}$ determines the agent's incentives for effort, and so the (IC) constraint (9) implies that effort solves

$$
\max _{a_{i}} y_{i i} a_{i}-\Psi\left(a_{i}\right)
$$

which is equivalent to $a_{i}=y_{i i} / k$. Finally, the sensitivity to peer output $y_{i j}$ is used to minimize the agent's exposure to systematic risk, and $y_{i i}<1$ reduces his exposure to project specific risk.

LEMMA 1 provides the standard contracting result that effort is attenuated relative to the first best $\left(a_{i}^{*}=y_{i i}^{*} / k<1 / k=a^{f b}\right)$ because of the cost of imposing risk on the agent. Effort decreases with the cost of effort $(k)$, the agent's risk aversion $(\lambda)$, and the residual risk $(\bar{\sigma})$. It 
also predicts that the relative sensitivity of the agent's wage to peer output versus his own output will correspond to the optimal benchmark in (12):

$$
\phi_{i}^{*} \equiv \sum_{j \in \mathcal{N}_{i}} \frac{y_{i j}^{*}}{y_{i i}^{*}}=-\bar{\theta} .
$$

Equation (17) forms the basis for standard tests of RPE in the empirical literature, which generally conclude that compensation tends to be much less negatively correlated with peer performance than is predicted by an optimal contracting framework. Indeed, many studies often find the opposite sign - pay is positively related to aggregate performance. A key goal of this paper is to understand how the optimal performance benchmark changes when agents have relative wage concerns, and how this change affects wages, productivity, and profits.

Remark. In the subsequent analysis, it will sometimes be useful to consider comparative statics in the size of the peer population, $n$, while keeping the fundamentals of the agency problem unchanged. To do so, we can vary $n$ while holding fixed $(\bar{\theta}, \bar{\sigma})$, which determine the information externalities, and let $\left(\rho, \sigma_{\epsilon}\right)$ adjust with $n$ according to (12) and (13). As shown in LEMMA 1, in the RPE setting the optimal actions $a_{i}$, relative contract sensitivities $\phi_{i}$, and profits $\pi_{I}$ (and thus total welfare), are all independent of $n$ given $(\bar{\theta}, \bar{\sigma})$.

\section{Peer-Contracting Equilibrium}

Now we consider the case in which agents have peer-dependent preferences $(\delta>0)$. Due to relative wage concerns, the agent is exposed to the output of all other agents both directly through his own contract as well as indirectly through his concern for other's wages. As illustrated in Figure 2, given a set of contracts with parameters $y_{i j}$, agent $i$ 's total exposure to output $q_{j}$ is given by ${ }^{17}$

$$
\beta_{i j} \equiv \frac{\partial c_{i}}{\partial q_{j}}=\frac{y_{i j}-\delta \sum_{k} \mu_{i k} y_{k j}}{1-\delta} .
$$

\footnotetext{
${ }^{17}$ We write $\Sigma_{k}$ as a shorthand for the summation is over all agents $k \in \mathcal{N}$.
} 
As equation (18) shows, agent $i$ has both a direct exposure (through his own contract) and an indirect exposure (through his concern for others' wages) to $j$ 's output, and hence there is now an interaction between the contracts offered to different agents. For example, the (IC) constraint (9) becomes

$$
\max _{a_{i}} \beta_{i i} a_{i}-\Psi\left(a_{i}\right) \Rightarrow a_{i}=\beta_{i i} / k
$$

Therefore, the agent's effort choice depends upon the contracts given to all agents. Similarly, the agent's risk is given by

$$
\operatorname{Var}\left(c_{i}\right)=\left[(1-\rho) \sum_{j} \beta_{i j}^{2}+\rho\left(\sum_{j} \beta_{i j}\right)^{2}\right] \sigma_{\epsilon}^{2},
$$

which again is a function of all contracts offered.

In the remainder of this section, we evaluate the consequences of this interdependency on equilibrium contracts. As we will see, we can restate the contracting problem for the principal in terms of the exposures $\beta$ in place of $y$, and then solve for the contract parameters that induce these exposures. First, we evaluate the consequences for efficiency, and show that a single principal can design contracts that hedge peer effects without sacrificing efficiency. When there are multiple principals, however, competition between them leads to a rat race effect in which agents work harder than in the RPE benchmark - and potentially harder than first best -- but firm profits and welfare are lower. We also look at the optimal wage sensitivities and correlations. There we show that, independent of welfare, peer effects lead to wage compression and, when sufficiently strong, cause wages to load positively on peer output.

\subsection{Efficiency and Rat Races}

\subsubsection{Optimal Welfare}

It is useful to begin by considering the case with a single team, or principal $(I=\mathcal{N})$. In that case the principal has full control of all contracts, and there is no issue of coordination. We can also view the single team case as the outcome a social planner would achieve given same information constraints faced by the principals. 
The following result demonstrates that we can restate the contracting problem for the principal in terms of the implied exposures $\beta$ in place of direct contract sensitivities $y$. More strikingly, despite the presence of peer effects, real outcomes and welfare are identical to the RPE setting.

Proposition I (Single Principal: Welfare Equivalence). Suppose agents have relative wage concerns $(\delta \geq 0)$ and there is a single principal $(I=\mathcal{N})$. Then the principal will choose $\beta$ to solve (14), and effort, expected output and profits are equal to the RPE outcome and independent of $\delta$. The optimal contracts are given by

$$
y^{S}=\Delta^{-1} y^{*}
$$

where $y^{S}, y^{*} \in \mathcal{R}^{N \times(N+1)}$ are matrices of contract sensitivities (with row $i$ the contract for agent $i$ ) and $\Delta \equiv \frac{1}{1-\delta}[I-\delta \mu]$ where $\mu$ is the $N \times N$ matrix of weights $\mu_{i j}$.

\section{ProOF: See Appendix.}

The key intuition behind Proposition I is that a single principal can effectively undo the peer effects in preferences via the wage contracts that are offered. As a result, effort and efficiency are unaffected by the strength $\delta$ of peer effects. Observed contracts and wages, however, will be affected by $\delta$ according to (21). Indeed, in this case the principal offers a wage that combines the RPE wage with a hedge position that insures against peer effects:

Corollary A (Optimal Wages). Let $w^{*}$ be the wages paid with RPE contracts, and let $w^{S}$ be the optimal wages with a single team. Then,

$$
w_{i}^{S}=w_{i}^{*}-\delta\left(w_{i}^{*}-w_{-i}^{S}\right)=(1-\delta) w_{i}^{*}+\delta w_{-i}^{S},
$$

and $\sum_{i} w_{i}^{S}=\sum_{i} w_{-i}^{S}=\sum_{i} w_{i}^{*}$

Proof: See Appendix. 
As the result shows, because the hedge positions aggregate to zero $\left(\sum_{i} w_{i}^{*}-w_{-i}^{S}=0\right)$, they can be implemented by the single principal at no cost. We will explore further the qualitative impacts on the observed contracts shortly, but first we consider the efficiency implications of having multiple teams. ${ }^{18}$

\subsubsection{Rat Race Distortions}

When there is more than one principal or team $(I \neq \mathcal{N})$, each principal must choose the contract for agents on her team while taking the contracts and actions of agents on other teams as given. In this case, there are two new effects that arise relative to the single principal case.

First, if the principal raises the expected wage of an agent on her team, she must also raise the wages of others on her team to compensate for the higher peer benchmark wage. But if her agents also have external peers on other teams, her impact on the peer benchmark is dampened, lowering the total cost of compensation for the principal. As a result, a principal whose agents have external peers is willing to increase incentives relative to the single principal case.

Second, by changing incentives and therefore the expected output of her own team, principal $I$ can influence the realized wages of agents on other teams whose contracts put weight on the output of team $I$. Then, by acting to lower the expected wage of other teams, the principal can raise the relative wage of agents on her own team. This negative externality provides an additional incentive to distort contracts.

We first consider the optimal contract for an individual team taking the contracts and action choices of other teams as given. To simplify the analysis and notation, we impose an additional restriction that all agents on team $I$ put the same aggregate weight $\mu_{I}$ on the wages of their teammates, and thus put total weight $1-\mu_{I}$ on non-teammates:

$$
\sum_{k \in I} \mu_{i k}=\mu_{I} \text { for all } i \in I
$$

\footnotetext{
${ }^{18}$ While we do not pursue it here, we can also solve for $w^{s}$ iteratively as the fixed point of the mapping defined by (22).
} 
LEMma 2 (TEAM CONTRACT). Given the contracts and actions of other teams, the optimal contract $y_{i}^{M}$ for agent $i$ on team $I$ has exposures $\beta_{i j}^{M}=\left(1+\alpha_{i}\right) y_{i j}^{*}$ and action $a_{i}^{M}=\left(1+\alpha_{i}\right) a_{i}^{*}$, where

$$
\alpha_{i} \equiv \frac{\delta}{1-\delta}\left(1-\mu_{I}-\sum_{\hat{i} \in I, j \notin I} \mu_{\hat{i} j} y_{j i}\right) .
$$

\section{Proof: See Appendix.}

The proof of LEMMA 2 relies on the ability of the principal for team $I$ to implement any set of exposures $\beta_{I}$ for her team members with an appropriate set of contracts $y_{I}$ (which will depend on both $\beta_{I}$ and the contracts $y_{J}$ chosen by all other teams). As a result, it is optimal for the principal to choose the relative exposures for agent $i, \beta_{i j} / \beta_{i k}$, to minimize risk as in the RPE case.

The effort choice, however, is distorted relative to the RPE or single principal setting due to the cross-team externalities described previously. This distortion is given by the factor $\alpha_{i}$ defined in (24). Note that if there are no peer effects $(\delta=0)$ or no external peers $\left(\mu_{I}=1\right.$, and therefore $\mu_{i k}=0$ for $\left.i \in I, k \notin I\right)$, then $\alpha_{i}=0$ and we achieve the same efficiency as the RPE benchmark. But if $\delta>0$ and $\mu_{I}<1$, the principal's effective cost of compensation is reduced, which raises the optimal effort choice. Second, if $y_{j i} \neq 0$, the principal can change agent $j$ 's expected wage by distorting agent $i$ 's effort. Then, by lowering $j$ 's expected wage, the principal lowers the cost of compensating any agent $\hat{i} \in I$ with $\mu_{\hat{i j}}>0$. The definition of $\alpha_{i}$ aggregates these effects.

While LEMMA 2 reveals the potential for effort to be distorted when there are multiple teams, the direction of the distortion is still unclear as it depends upon the equilibrium contracts used by all teams. That is, the above result provides the optimal response for one team given the contracts of other teams. In equilibrium, all teams choose optimal responses to each other. We 
evaluate this equilibrium next, and demonstrate that in fact the distortion leads to higher effort than the second-best setting, and, for $\delta$ close to 1 , it will even exceed the first best effort level.

For tractability, we make additional symmetry assumptions to compute the equilibrium. First, each team has the same size:

$$
N_{I}=N_{\hat{I}} \text { for all teams } I, \hat{I} \text {. }
$$

Second, agents place the same weight on peers who are external to their team:

$$
\mu_{i j}=\mu_{e}=\frac{1-\mu_{I}}{N-N_{I}} \text {, for all } i \in I \text { and } j \notin I .
$$

Note that we allow the internal weights within teams to be different, though the aggregate internal weight $\mu_{I}$ remains constant. Despite that, these conditions are sufficient to imply that the equilibrium effort distortions will be symmetric (even though contracts need not be). Our next result characterizes this outcome, and demonstrates a rat race effect in which the effort of each agent is distorted upward $(\alpha>0)$, reducing overall efficiency.

Proposition II (Symmetric Team Equilibrium: Rat Race). In the multi-team equilibrium, optimal contracts $\left\{y_{i j}^{M}\right\}_{j>0}$ and actions $a_{i}^{M}$ are given by

$$
y_{i j}^{M}=(1+\alpha) y_{i j}^{S} \text { and } a_{i}^{M}=(1+\alpha) a_{i}^{*},
$$

where

$$
1+\alpha=\frac{1}{1-\delta\left(\frac{1-\mu_{I}}{1-\delta \mu_{I}}\right)\left(1-N_{I} y_{e}^{S}\right)} \geq 1
$$

and $y_{i j}^{S}$ follows from (21), and for external peers is given by

$$
y_{e}^{S}=\frac{\delta \mu_{e}(1-\bar{\theta})-\frac{1}{n} \bar{\theta}(1-\delta)}{1-\delta+N \delta \mu_{e}} y_{i i}^{*}<\frac{1}{N} .
$$

If $\delta>0$ and $\mu_{I}<1$, then $\alpha>0$. Expected wages and profits per agent are given by 


$$
E\left[w_{i}^{M}\right]=c_{0}+\frac{1}{2} a_{i}^{*}(1+\alpha)^{2} \text { and } \pi_{i}^{M}=\pi_{i}^{*}-\frac{1}{2} a_{i}^{*} \alpha^{2}
$$

Proof: See Appendix.

Proposition II establishes that when principals compete with each other, they neglect the peer externality on other teams and thus provide inefficiently high-powered incentives, with contract terms and effort scaled by the factor $(1+\alpha)$. Agent's work harder, but the increase in the expected wage that is required more than offsets the increase in productivity, and the expected profit of each team is lower than in the RPE or single team benchmark. The next result demonstrates that these distortions increase with the strength of external peer-effects - due to a higher number or higher weight on external peers - and can cause effort to exceed even the firstbest level.

Proposition III (RAt Race Monotonicity). In the team equilibrium with multiple teams and positive external weights $\mu_{e}>0$, the rate race distortion $\alpha$ decreases with team size $N_{I}$ and increases with the external weights $\mu_{e}$ and the strength $\delta$ of peer effects. As $\delta \rightarrow 1$, equilibrium effort increases and ultimately exceeds the first-best level:

$$
(1+\alpha) a^{*} \rightarrow \frac{N}{N_{I}(1-\bar{\theta})} \frac{1}{k}>\frac{1}{k}=a^{f b}
$$

\section{Proof: See Appendix.}

The following result provides an even simpler characterization of the magnitude of the rat race effect when the number of teams grows large, and shows that the strength of the effect increases with both $\delta$ and the total weight $1-\mu_{I}$ put on external peers:

Corollary B (Diffuse External Peers). Holding $\left(N_{I}, \mu_{I}\right)$ fixed as $N \rightarrow \infty$, $\alpha \rightarrow \frac{\delta}{1-\delta}\left(1-\mu_{I}\right)$. When $N$ is large, $\alpha$ increases with $N$ if and only if $\frac{\delta-\bar{\theta}}{\delta(1-\bar{\theta})}>\mu_{I}$. 
ProOF: See Appendix.

COROLlaRY B also shows the despite the constant returns to scale in our model, we may see either increasing or decreasing returns to scale depending on the magnitude of $\delta$ versus $\mu_{I}$.

We will discuss these results further in Section 5.2 when we consider the potential consequences of public disclosure of wage contracts.

While our prior results have focused on symmetrically sized teams, we can also apply LEMMa 2 to evaluate equilibria when teams are asymmetric. The following result considers the case with two teams of differing size. Because each principal does not take into account the effect of its wage contracts on the other team, the rat race externality is largest for the smaller team. As a result, agents on the smaller team will work harder, but despite having higher productivity, its per capita expected profits are lower.

Proposition IV (Asymmetric Team Size). Suppose there are two teams $I$ and $J$ of size $1<N_{I}<N_{J}$, with symmetric external weights $\mu_{i j}=\mu_{e}<1 / N$ for $i \in I$ and $j \in J$. Then for $\delta \in(0,0.8)$, the rat race effect is larger for the smaller team, $\alpha_{i}>\left|\alpha_{j}\right|$, and its expected profits are lower.

\section{ProOF: See Appendix.}

The intuition for this result is that the rat race externality is strongest when the size of the team is small. If the size of the team is very large (close to $N$ ) then the rat race externality is weak. Moreover, if $\delta>0.5$ and $y_{e}^{S}$ is also large, it is possible that $\alpha_{J}<0$; in that case the principal of the large team reduces effort so as not to indirectly benefit external agents and raise her own cost of compensation.

Finally for $\delta$ very close to 1 , the rat race distortion can become so large that at least one team becomes unprofitable (see (30)). In that case, we may expect principals to exit and the remaining teams to grow, creating an endogenous restriction on the number and size of teams that can participate in an industry before the rat race effect makes it unprofitable. 


\subsection{Wage Sensitivity and Correlation}

The prior results have examined the welfare consequences of peer effects. In this section we consider their effects on observed contracts and wages. A key insight behind Proposition I and Proposition II is that each principal can effectively undo the peer effects in preferences via the wage contracts that are offered. By hedging the peer preferences via the contract, the principal can provide a relative exposure $\beta_{i}$ to agent $i$ that exposes him to the same level of risk $\bar{\sigma}$ per incremental unit of output as in the RPE contract. While the level of output is impacted by the rat race effect, optimal risk sharing is maintained.

But while the implied total exposures match the RPE outcome, the observed contracts $y_{i}$ will be affected by the strength $\delta$ of relative wage concerns. As $\delta$ increases and peer effects become more dominant, contracts will be distorted more and more in order to hedge agents against relative wage shocks. In this section we explore the consequences of these distortions and their implications for wage sensitivity and correlation.

Recall that from Proposition I, the optimal single-team contracts are given by $y^{S}=\Delta^{-1} y^{*}$, where $\Delta \equiv(1-\delta)^{-1}[I-\delta \mu]$ is the peer-weighting matrix and $y^{*}$ is the optimal RPE contract. Proposition II further shows that when there are multiple teams the only change to the optimal contract loadings is a rescaling by the rat race factor $(1+\alpha)$, and hence relative contract sensitivities are unchanged. We can now use these results to further analyze the impact of peereffects on optimal contracts:

Proposition V (Contract Sensitivities). In the single team setting of Proposition I, contract loadings are compressed relative to the RPE setting:

$$
y_{i i}^{*} \geq y_{i i}^{S} \geq y_{i j}^{S} \geq y_{i j}^{*}=-\frac{1}{n} \bar{\theta} y_{i i}^{*} .
$$

With single or multiple teams (as in Proposition II), agent $i$ 's sensitivity to peer output in the optimal contract satisfies

$$
\phi_{i}=\phi_{i}^{M}=\phi_{i}^{S} \equiv \sum_{j \in \mathcal{N}_{i}} \frac{y_{i j}^{S}}{y_{i i}^{S}}=\frac{1-\bar{\theta}}{\left(1+\frac{1}{n} \bar{\theta}\right) \Delta_{i i}^{-1}-\frac{1}{n} \bar{\theta}}-1 .
$$


When there are no peer effects $(\delta=0), \phi_{i}=-\bar{\theta}$. But as $\delta$ increases, $\phi_{i}$ strictly increases, and as $\delta \rightarrow 1$,

$$
\frac{y_{i j}^{S}}{y_{i i}^{S}} \rightarrow 1 \text { for all } j, \text { and } \phi_{i} \rightarrow n
$$

Proof: See Appendix.

Proposition V demonstrates that peer effects compress the contract loadings and increase the relative importance of peer output in the wage determination, with the overall peer sensitivity depending solely on the diagonal term $\Delta_{i i}^{-1}$ of the inverted peer-weighting matrix. Most strikingly, as $\delta \rightarrow 1$ and peer effects dominate, the principal will optimally adjust contracts to have equal weight on each agent's output. In other words, agents will be compensated based on aggregate output only, and wages will become perfectly correlated $\left(w_{i}^{M}=w_{-i}^{M}=w_{j}^{M}\right) .{ }^{19}$ By doing so, "relative wage risk" is eliminated.

Yet despite this apparent distortion in contracts, in the single-team case effort levels are maintained and the principal's expected profit is unchanged. This result highlights that fact that even if contracts appear inefficient relative to RPE, such contracts may be optimal and efficient if agents have peer-dependent preferences. The inefficiencies that do arise are the result of rat race externalities across teams and are not due to failures of RPE.

\subsection{Special Cases}

Thus far we have made no assumptions on the precise network structure of preferences embodied in $\mu$. In this section we consider several special cases of peer weights and compute explicit solutions for the optimal contracts and their sensitivities. We begin with a setting of uniform internal weights and external weights. Specifically, in addition to (25) and (26), we assume agent's put the same weight $\mu_{t}$ on each teammate:

\footnotetext{
${ }^{19}$ In the single principal case, this result is immediate from COROLLARY A. In the case of multiple teams, wages are rescaled due to rat race effects.
} 


$$
\mu_{i \hat{i}}=\mu_{t}=\frac{\mu_{I}}{N_{I}-1}=\frac{1-\left(N-N_{I}\right) \mu_{e}}{N_{I}-1} \text { for all } i, \hat{i} \in I \text { with } i \neq \hat{i}
$$

It is natural to assume agents put more weight on internal versus external peers; the following result demonstrates that same weighting will be inherited by equilibrium contracts.

Proposition VI (Uniform Internal AND External Weights). Suppose agents have uniform internal and external weights with $\mu_{t} \geq 1 / n \geq \mu_{e}$. Then equilibrium contracts have uniform internal and external exposures

$$
y_{t} \geq y_{e}=(1+\alpha) \frac{\delta \mu_{e}(1-\bar{\theta})-\frac{1}{n} \bar{\theta}(1-\delta)}{1-\delta+N \delta \mu_{e}} y_{i i}^{*}
$$

with equality if and only if $\mu_{e}=\mu_{t}=1 / n$ or $\delta=0$. Holding team size fixed, $\phi_{i}$ and $y_{e}$ are increasing in $\mu_{e}$, with

$$
y_{e} \geq 0 \text { if and only if } \frac{\delta}{1-\delta} \geq \frac{1}{n \mu_{e}}\left(\frac{\bar{\theta}}{1-\bar{\theta}}\right)
$$

Proof: See Appendix.

Proposition VI shows that with uniform weights, wages will depend on the agent's own output, team output, and aggregate output. The exposure to aggregate output, $y_{e}$, is positive for $\delta$ sufficiently high, while the incremental loading on team output, $y_{t}-y_{e}$, is always nonnegative.

The following corollary examines the special case of equal weights, which also includes the setting with unitary teams.

Corollary C (Equal Weights). Suppose $\mu_{i j}=1 / n$ for all $i \neq j$. Then

$$
y_{i i}^{S}=y_{i i}^{*}\left[1-\delta\left(\frac{n+\bar{\theta}}{n+\delta}\right)\right], y_{t}^{S}=y_{e}^{S}=y_{i i}^{*}\left(\frac{\delta-\bar{\theta}}{n+\delta}\right) \text {, and } \phi_{i}=\frac{n(\delta-\bar{\theta})}{n(1-\delta)+\delta(1-\bar{\theta})} \text {. }
$$

Finally, with unitary teams $\left(N_{I}=1\right)$, the rat race factor is 


$$
1+\alpha=\frac{1}{1-\delta+\delta y_{e}^{S}}=\frac{1}{1-\delta+\delta\left(\frac{\delta-\bar{\theta}}{n+\delta}\right) y_{i i}^{*}}
$$

Proof: See Appendix.

Again, consistent with Proposition III and Proposition V, we can see easily from (38) and (39) that as $\delta$ increases optimal contracts put more weight on peer performance and less weight on the agent's own performance, and the rat race effect intensifies.

Figure 1 shows the impact of peer effects on wage volatility and correlation in the case with uniform weights. Note that wage volatility decreases, and wage correlation increases and converges to 1 , as $\delta$ increases. ${ }^{20}$

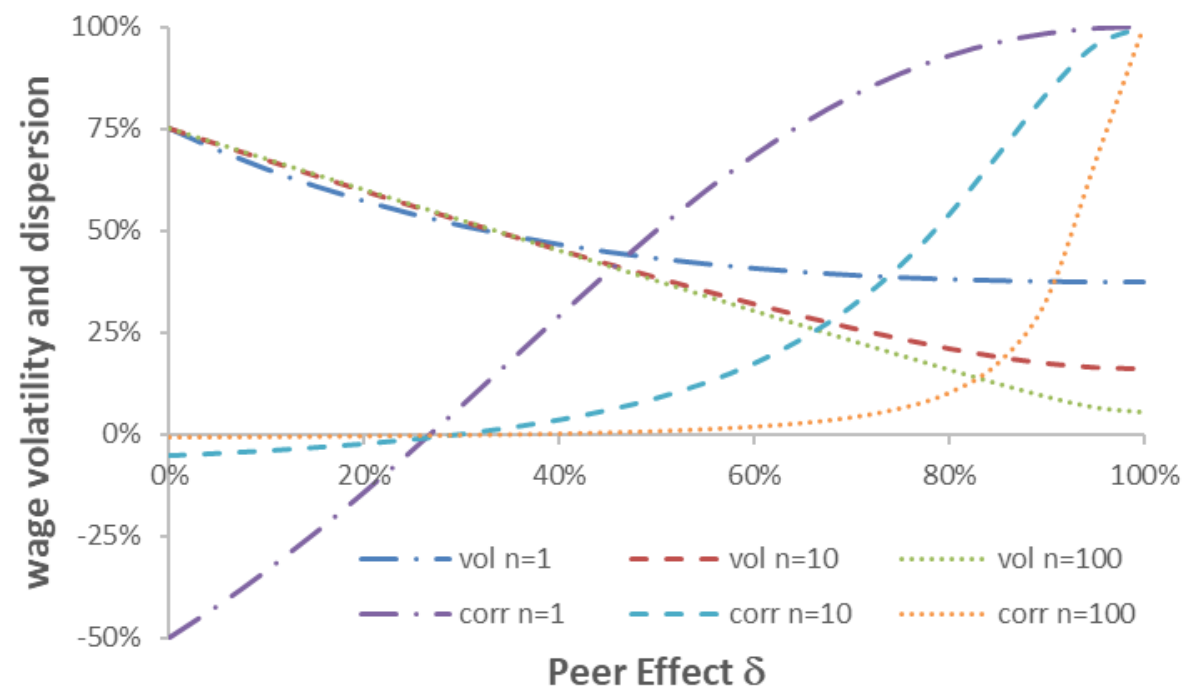

Figure 3: Wage Volatility and Dispersion

Wage volatility (expressed relative to $(1+\alpha) \bar{\sigma}$ ) declines, and wage correlation increases (converging to 1 ), with the strength $\delta$ of peer effects. (Uniform weights, $\bar{\theta}=50 \%, y_{i i}^{*}=75 \%$ ).

\footnotetext{
${ }^{20}$ Englmaier and Wambach (2010) demonstrate that wage compression results if agents are inequity averse, and thus suffer a disutility if their payoff exceeds that of others. Here, even though agents gain from being ahead of their peers, it is still optimal to reduce wage variation.
} 
COROLlary C implies that with equal weights, wage contracts will load positively on peer output as long as $\delta>\bar{\theta}$, in stark contrast to the RPE prediction. The next result demonstrates that this same conclusion holds more generally as long as the set of peers is large, and the weights agents put on others are diffuse.

Corollary D (Diffuse Weights). Suppose $\|\mu\|_{\max } \rightarrow 0$ as $N \rightarrow \infty$. Then

$$
\phi_{i}^{S} \rightarrow \frac{\delta-\bar{\theta}}{1-\delta} \text { as } N \rightarrow \infty .
$$

ProOF: See Appendix.

As a contrast to the case of diffuse weights, we consider next a case with concentrated weights. Suppose agents are arranged in a circle and care only about their nearest neighbors; that is $\mu_{i j}=\frac{1}{2}$ if and only if $|i-j| \in\{1, n\}$. We can again show that the total weight on peers is positive as long as the RPE hedge ratio $\bar{\theta}$ is not too large relative to $\delta$ (though the constraint is a bit tighter than in Proposition VI). In addition, while the agent only cares directly about his immediate neighbors, the contract loading for every peer is distorted above the RPE case, with the distortion decreasing with distance to that peer. The reason the contract is distorted even for non-neighboring peers is due to a chain reaction effect through the network of peer relationships.

Corollary E (Circular Weights). Consider a circular network of peers in which each agent puts equal weight on their two nearest neighbors. Then $y_{i j}$ is decreasing in the distance between $i$ and $j$, with $\min _{j} y_{i j} / y_{i i}>-\frac{1}{n} \bar{\theta}$. Moreover,

$$
\lim _{N \rightarrow \infty} \phi_{i}>0 \text { iff } \bar{\theta}<1-\sqrt{\frac{1-\delta}{1+\delta}} .
$$

\section{ProOF: See Appendix.}

As mentioned in Section 2.1, our general formulation of peer-based preferences allows $\mu_{i i}>0$, so that the agent puts weight on his own wage in the benchmark. Raising $\mu_{i i}$, therefore, 
effectively lowers the strength of the agent's relative wage concerns (see footnote 11). The following result shows that if we increase the weight agent $i$ puts on his own wage, and reduce the weight he puts on his peers, then his relative sensitivity to peer output in the optimal contract declines.

COROLlaRy F (ASYMmetric Delta). Let $\mu(\lambda)$ be a valid peer matrix parameterized by $\lambda$. Suppose $\mu_{i i}^{\prime}(\lambda)>0$ and $\mu_{i j}^{\prime}(\lambda) \leq 0$ for all $j \neq i$. Then $\phi_{i}^{\prime}(\lambda)<0$.

Proof: See Appendix.

\section{Asymmetric Visibility and Efficiency Gains}

Our prior results demonstrate that profits either stay the same (with a single principal) or decline (with multiple principals), with the strength peer effects when peer relationships are symmetric. In this section we relax the symmetry requirement and show that in when some peers are "more visible" than others, the principal can exploit this asymmetry and increase profits.

Specifically, if we define the "visibility" $\bar{\mu}_{j}$ of agent $j$ as the collective weight put on that agent by others, then under our current assumption (1) we have:

$$
\bar{\mu}_{j} \equiv \sum_{i} \mu_{i j}=1 \text { for all } j
$$

That is, each agent has symmetric visibility (equivalently, in mathematical terms, the matrix $\mu$ is doubly stochastic). Indeed, it is straightforward to show that our welfare results in Section 3.1 rely only on (40), and not on the stronger pairwise symmetry assumption in (1). ${ }^{21}$

Now suppose we relax (40), so that some agents are more visible than others. As before, the principal can still use the agent's wage contract to hedge peer effects. In addition, however, the principal may exploit the asymmetric importance of agents and provide higher incentives (and therefore high expected compensation) to agents who are less visible, and lower the wages and

\footnotetext{
${ }^{21}$ See e.g. the proof of Proposition I, which only uses symmetry to establish that $1^{\prime} \Delta^{-1}=1^{\prime} \mu=1^{\prime}$.
} 
incentives to agents who are highly visible, in a way that lowers the expected cost of compensation and raises profits.

Proposition VII (Asymmetric Visibility and EfFiciency Gains). Suppose that we relax the symmetry assumption so that for at least one agent $j, \bar{\mu}_{j} \neq 1$. If $\delta>0$, then although the relative contract sensitivities satisfy (33) as before, a single principal can distort effort to achieve higher expected profits than in the RPE case $(\delta=0)$. Then the relative cost of compensating agent $j$ is given by

$$
\omega_{j} \equiv \sum_{i} \Delta_{i j}^{-1}, \text { where } \frac{1}{N} \sum_{j} \omega_{j}=1<\frac{1}{N} \sum_{j} \omega_{j}^{-1},
$$

and the equilibrium effort provision and the expected profit from each agent is asymmetric with

$$
a_{j}^{A}=\omega_{j}^{-1} a_{j}^{*} \text { and } \pi_{j}^{A}=q_{0}+\omega_{j}^{-1}\left(\frac{1}{2} a_{i}^{*}\right)-c_{0}=\pi_{j}^{*}+\frac{1}{2}\left(\omega_{j}^{-1}-1\right) a_{i}^{*}
$$

\section{Proof: See Appendix.}

We can interpret $\omega_{j}$ as the effective cost of compensating agent $j$ once we account for the influence of his wage on the utility of his peers. (Recall from Proposition I that $\Delta^{-1}$ determines the contract adjustments required for the principal to hedge each agent's exposure to his peer benchmark.) While the average $\omega_{j}$ is 1 (because $1^{\prime} \Delta^{-1} 1=1^{\prime} 1=N$ ), when visibility $\bar{\mu}_{j}$ is asymmetric so is the effective cost. The proposition shows that in that case, the principal can lower the total cost of compensation by shifting incentives (and hence wages) to those agents with the lowest effective cost.

To illustrate the relationship between the preference weights $(\mu, \delta)$ and the principal's expected profit, Figure 4 shows the average benefit per agent, $\left(\frac{1}{N} \sum_{j} \omega_{j}^{-1}\right)-1$, for a range of randomly generated models. Here, we have measured the asymmetry in $\mu$ according to the heterogeneity in the agents' visibility $\bar{\mu}$. As the figure shows, the principal's expected profit increases with this variation (standard deviation of $\bar{\mu}_{j}$ ) as well as with the intensity $\delta$ of the peer 
effects. The latter effect, monotonicity with respect to $\delta$ for a given $\mu$, appears to hold quite generally across all numerical examples.

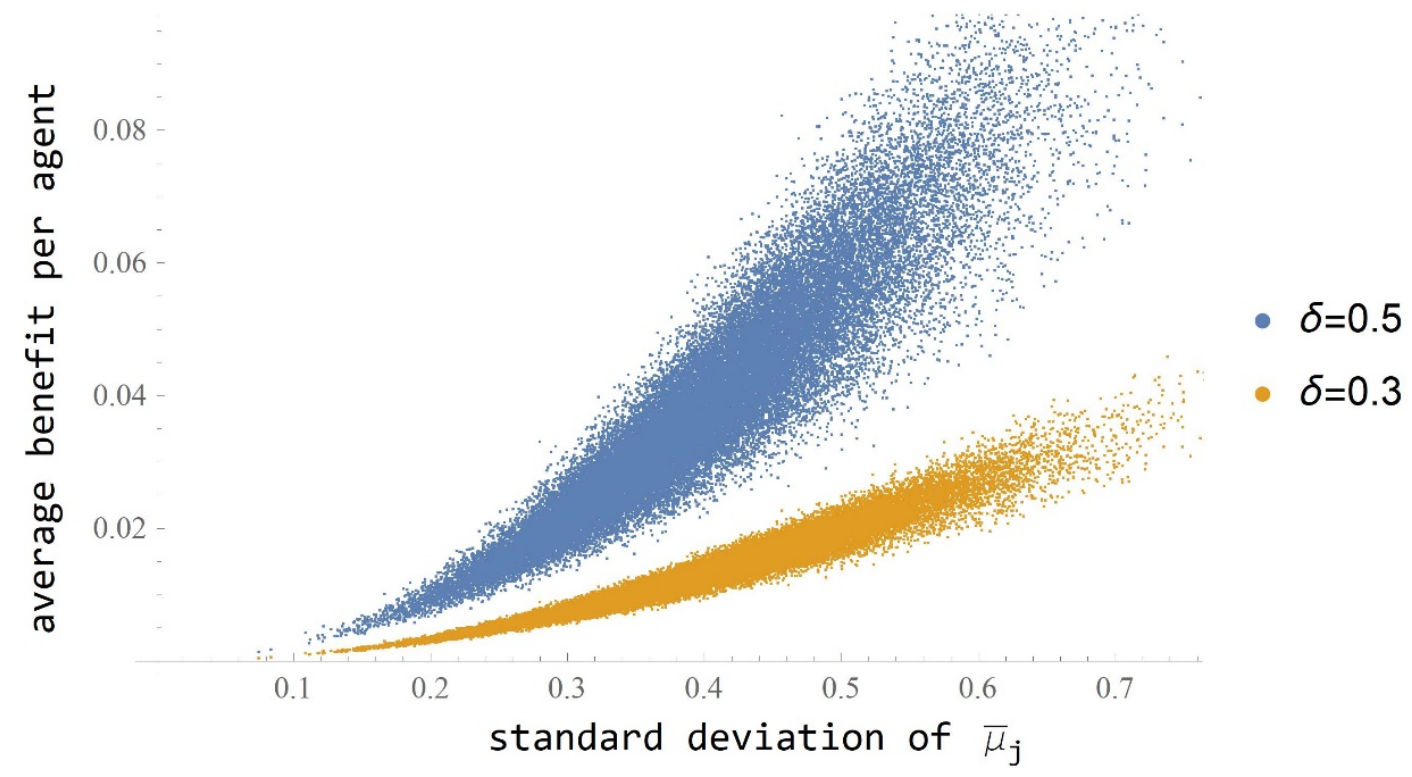

Figure 4: Benefit to Principal from Agent Heterogeneity

The average benefit per agent to the principal $\frac{1}{N} \sum_{j} \omega_{j}^{-1}-1$ as function of standard deviation of $\bar{\mu}_{j}$. Simulating 40,000 random stochastic matrixes $\mu$ for $N=10$. Blue (orange) dots are for $\delta=0.3(\delta=0.5)$.

To further elucidate the source of increased profits to the principal, we next consider a specific natural example of asymmetry in which there are two types of agents:

- Independent Agents: $\mu_{j j}=1, \mu_{j k}=0$ for $k \neq j$; thus their effective $\delta$ is zero. These agents care only about their own wage, and do not care about the wages of their peers.

- Peer-Dependent Agents: $\delta>0, \mu_{m m}=0, \mu_{m k}=1 / n$ for $k \neq m$. These agents benchmark their wage to the average of their peers.

Note that in this example, because peer-dependent agents care about independent agents, but not vice versa, independent agents are more visible. The following proposition shows that as a result, 
independent agents will be given lower equilibrium incentives and wages. We also show that the principal's profit increases with the heterogeneity of the population.

Proposition VIII (Mixed Population). Suppose agents $j \in J$ are independent, whereas agents $m \notin J$ are peer-dependent. Then independent agents receive a standard RPE contract with $\phi_{j}^{A}=-\bar{\theta}$. Peer-dependent agents work harder than independent agents,

$$
\frac{a_{m}^{A}}{a_{j}^{A}}=\frac{1+\frac{1}{n} \delta}{1-\delta} \equiv \psi>1
$$

and furthermore,

$$
\frac{1}{N} \sum_{j} \omega_{j}^{-1}=1+\left(\psi+\psi^{-1}-2\right) \frac{|J|}{N}\left(1-\frac{|J|}{N}\right),
$$

so that the principal's profit per agent increases with an increase in population heterogeneity: that is, with an increase in $\delta$, or shifting the proportion of independent agents closer to $50 \%$.

Proof: See Appendix.

Note that for $|J|=\frac{1}{2} N$, the principal's gain per agent decreases in $N$ with the limit

$$
\frac{1}{N} \sum_{j}\left(\omega_{j}^{-1}-1\right) \rightarrow \frac{\delta^{2}}{4(1-\delta)} \text { as } N \rightarrow \infty
$$

Thus, the average gain per agent does not completely dissipate even when $N$ is very large.

The ability of the single principal to internalize the impact of the contracts he sets allows him to capitalize on population heterogeneity. With multiple principals, there will be competing effects between the population heterogeneity within teams, which will increase efficiency, and the rat-race effect across teams, which will decrease efficiency. 


\section{Disclosure Effects}

In the standard RPE contracting framework without peer effects, agent's incentives are independent of each other's contracts. As a result, the timing and visibility of contracts and wages are not consequential. As we show in this section, however, with peer effects these details will matter. For example, if principals can negotiate privately with individual agents, additional rat race effects emerge within teams. In addition, the public disclosure of wage contracts across teams may increase their salience, strengthening peer effects, while also creating incentives for principals to distort contracts to affect the productivity of other teams.

\subsection{Private Negotiation}

Thus far we have assumed that each principal discloses to her team the incentive contracts to be used within the firm. This assumption implies that the principal cannot privately negotiate (or renegotiate) these contracts with individual agents. If instead individual contracts terms can be set or altered in a way that is hidden from other team members, then in equilibrium we should require that contracts be "renegotiation-proof" with respect to any principal-agent pair. That is, in equilibrium, there should be no alternative contract the principal could offer to a single agent which the agent would accept and would raise the principal's expected profit, while holding other contracts and effort as given.

When privately negotiating, the principal and agent will ignore the impact of their wage choice on the utility of other agents, as well as try to lower the wage of others through the performance benchmark, just as in the setting of multiple teams discussed earlier. Moreover, there is now an added benefit to the principal: changing the agent's effort in a way that lowers the wage of other agents within the same team contributes directly to the principal's profits.

Though there is an incentive to renegotiate, the opportunity to do so must hurt the principal ex-ante. In equilibrium, other agents within the team will anticipate the renegotiated contract and seek commensurate terms. In other words, because the renegotiation-proof contract could always be proposed in an environment with disclosure, allowing hidden renegotiation only constrains the principal. But while each principal is individually worse off with hidden contracting, the 
equilibrium consequence of renegotiation is less clear, as constraining contracts in this way might reduce some of the "rat race" inefficiency that arises with multiple teams.

The following result characterizes the optimal contract when contracts are privately negotiated:

Lemma 3 (Private Negotiation Contract). Given the contracts and actions of other agents, principal $I$ has an incentive to renegotiate privately with agent $i$ unless agent $i$ has exposure $\beta_{i}^{R}=\left(1+\alpha_{i}^{R}\right) y_{i}^{*}$ where

$$
1+\alpha_{i}^{R} \equiv\left(\frac{1-\delta \mu_{i i}}{1-\delta}\right)\left(1-\frac{\delta}{1-\delta \mu_{i i}} \sum_{j \neq i} \mu_{i j} y_{j i}-\sum_{\hat{i} \in I_{i}} y_{\hat{i} i}\right)
$$

\section{Proof: See Appendix.}

To understand (44), it is useful to consider the case with $\mu_{i i}=0$. (Recall that $\mu_{i i}>0$ is equivalent to adjusting $\delta$ for agent $i$, see footnote 11). In that case,

$$
1+\alpha_{i}^{R} \equiv\left(\frac{1}{1-\delta}\right)\left(1-\delta \sum_{j \neq i} \mu_{i j} y_{j i}-\sum_{\hat{i} \in I_{i}} y_{\hat{i} i}\right)
$$

Here the term $\sum_{j \neq i} \mu_{i j} y_{j i}$ captures the benefit to agent $i$ from altering agent $j$ 's expected wage. The term $\sum_{\hat{i} \in I_{i}} y_{\hat{i} i}$ captures the benefit to the principal from altering the expected wages of other agents on team $I$. Finally, we note that (24) and (44) coincide in the case of a single agent team, as in that case the contract is already negotiated in isolation.

To evaluate the equilibrium impact of renegotiation, we again consider the symmetric case, and set $\mu_{i i}=0$ for simplicity.

Proposition IX (Private Negotiation Equilibrium). Under (25) and (26), with $\mu_{i i}=0$ for all $i$, in the symmetric equilibrium with private renegotiation, 


$$
1+\alpha^{R} \equiv \frac{1}{1-\delta+(\delta-\bar{\theta}) y_{i i}^{*}-\left(N-N_{I}\right) y_{e}^{S}} \geq 1+\alpha,
$$

with equality only if $N_{I}=1$. If all weights are equal $\left(\mu_{i j}=1 / n\right)$, then letting $n_{I}=N_{I}-1$,

$$
1+\alpha^{R}=\frac{1}{1-\delta+\left(n_{I}+\delta\right) y_{e}^{S}}=\frac{1}{1-\delta+\left(\frac{n_{I}+\delta}{n+\delta}\right)(\delta-\bar{\theta}) y_{i i}^{*}} .
$$

Proof: See Appendix.

Note that in the case of a single principal $\left(N=N_{I}\right)$, (46) implies

$$
1+\alpha^{R}=\frac{1}{1-\delta+(\delta-\bar{\theta}) y_{i i}^{*}}>1
$$

Therefore, renegotiation leads to higher expected output and lower expected profit for the principal. On the other hand, with unitary teams $\left(n_{I}=0\right),(47)$ implies

$$
1+\alpha^{R}=\frac{1}{1-\delta+\delta y_{e}^{S}}=\frac{1}{1-\delta+(\delta-\bar{\theta})\left(\frac{\delta}{n+\delta}\right) y_{i i}^{*}},
$$

which matches the previous outcome in PrOPOSITION VI without renegotiation - again, with single-agent teams each principal is already negotiating in private and there is no scope for further renegotiation. Comparing (48) and (49), we see that for $\delta<\bar{\theta}$, the rat race effect is even stronger for a single principal who can privately negotiate than it is for independent principals with singleton teams.

\subsection{Wage Transparency and Public Disclosure}

Recent regulation has increased disclosure requirements regarding executive compensation. The SEC now requires compensation disclosures for the CEO, CFO, and the three additional most highly compensated officers the firm. Websites such as Glassdoor collect and provide data on salaries for a broad range of managerial positions within firms. Compensation 
disclosure requirements for public employees have also increased as a result of transparency and accountability measures. For example, individual faculty member salaries for all faculty at the University of California can be looked up online.

In the context of our model, we interpret greater wage transparency and disclosure as leading to an increase in the salience of peer compensation. ${ }^{22}$ As a result, individuals are likely to put higher weight on their relative, versus absolute, wage - that is, an increase in $\delta$ - as well as put higher weight on more distant peers - an increase in $\mu_{e}$. These effects may be due to the agent's own visibility of others' wages, or the fact that agents believe that others can more easily make such comparisons. ${ }^{23}$

Under this interpretation, the results of Section 3 allow for the following empirical implications of increased disclosure:

Corollary G (Wage Transparency). In the symmetric team setting of Proposition II, suppose an increase in transparency or disclosure leads to an increase in $\delta$. This change will lead to an

- Increase in rat race effects $(\alpha)$ : higher wages and productivity, lower profits,

- Increase in the wage contract exposure to external peers $\left(y_{e}\right)$,

- Increase in wage correlation and sensitivity to peer output $(\phi)$.

The same results apply for an increase in $\mu_{e}$, where in the case of the wage correlation and peer sensitivity we additionally assume uniform internal weights.

Proof: See Appendix.

The effects of increasing $\delta$ and $\mu_{e}$ are illustrated in Figure 5. Note that an increase in $\mu_{e}$, holding $N_{I}$ and $N$ fixed, necessitates a decline in $\mu_{t}$, which explains the decline in $y_{t}^{S}$ in the right panel.

\footnotetext{
${ }^{22}$ Card et.al. (2012) provide field-based confirmation of relative pay comparisons by randomized manipulation of revelation of information on coworkers' salaries for University of California employees.

${ }^{23}$ For example, we can think of the weights $\mu_{i j}$ as based in part on the probability that others are able to see and compare agent $i$ and $j$ 's wages.
} 


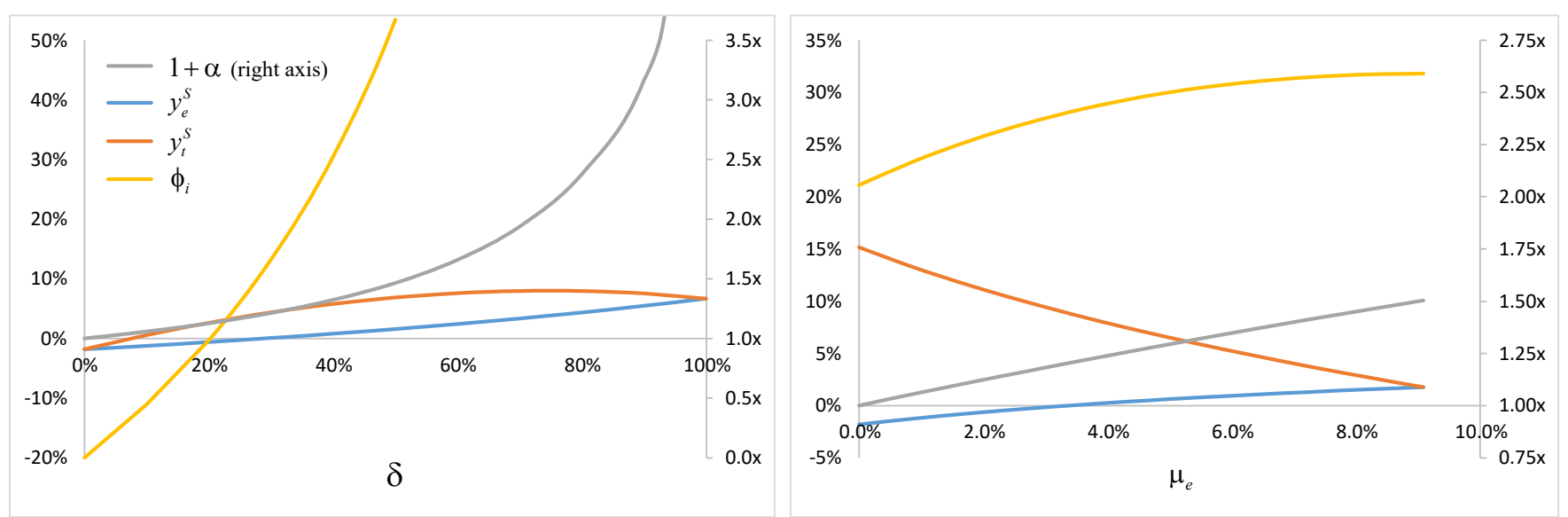

Figure 5: Effect of Increased Wage Transparency on Contract Sensitivities and Rat Race Effects Increased wage transparency strengthens peer effects (higher $\delta$ ) especially for external peers (higher $\mu_{e}$ ). As a consequences, the contract sensitivities and rat race effects increase.

(Parameters: $N=12, N_{t}=3, \bar{\theta}=20 \%, \delta=40 \%, \mu_{e}=5.6 \%, \mu_{t}=50 \%$ ).

Recent regulation has increased disclosure requirements of CEO compensation. Our results suggest this change should lead to higher expected compensation. Consistent with this prediction, Gipper (2016) shows that the 2006 SEC enhanced disclosure requirements ${ }^{24}$ on managerial compensation increased CEOs' pay. In addition, the U.S Congress Jumpstart Our Business Startups (JOBS) Act of 2012, that rolled back some disclosure requirements for a subset of firms lead to a decline in managers' compensation. ${ }^{25}$

\subsection{Ex Ante Contract Disclosure}

We have assumed thus far that agents can only see the contracts or wages of other teams after making their effort decisions. In this section we consider an additional effect that arises if contracts are disclosed across teams before effort is determined. Specifically, we modify the timing in Figure 1 so that prior to choosing effort, each agent can observe the wage contracts of agents on other teams.

\footnotetext{
${ }^{24}$ For details see http://www.sec.gov/rules/final/2006/33-8732a.pdf.

${ }^{25}$ Further evidence, in other settings, that increased disclosure of executive compensation increases executive pay is provided in Park, Nelson, and Huson (2001), Perry, and Zenner (2001), Schmidt (2012), and Mas (2016).
} 


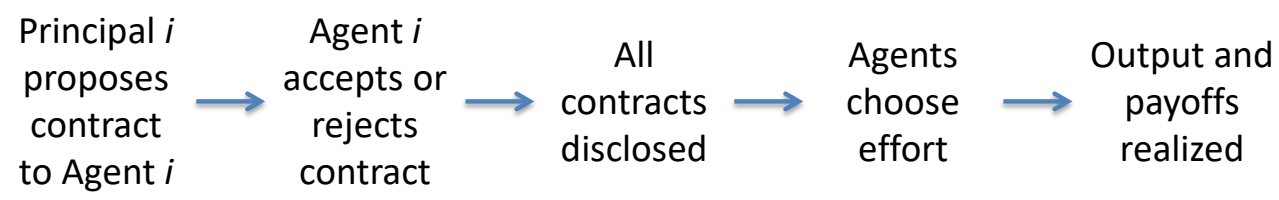

Figure 6: Contracting Game with Ex Ante Disclosure

Ex ante disclosure matters because agent $i \in I$ 's contract, once revealed, will influence the effort choice of other agents $j \notin I$. For example, an increase in $y_{i j}$, the loading of $i$ 's contract on $j$ 's output, will reduce agent $j$ 's effort incentives, since higher $q_{j}$ would have a more positive impact on $i$ 's wage, reducing $j$ 's utility. Principal $I$ can take advantage of this effect, by offering agent $i$ a contract with higher $y_{i j}$, and then, anticipating that agent $j$ will reduce effort and earn a lower wage as a result, lower the expected wage paid to team $I$ (given the lower expected peer benchmark).

This intuition has several important implications. First, it suggests that each principal will no longer choose the implied sensitivity to minimize the agent's risk. Instead, the principal will raise the sensitivity to external peers in order to distort their effort downward. But, because the agent is now exposed to additional external output risk, the contract will expose him to less output risk from his own team. Finally, because these distortions raise the cost of providing incentives, the optimal level of effort declines.

The following result confirms these effects. For simplicity, we focus on the case with uniform internal and external weights, where those weights may differ from each other. We show that in the optimal contract the agent's effort (determined by $\beta_{i i}^{D}$ ) is distorted downward, as is the agent's implied exposure to the output of his teammates $\left(\beta_{i \hat{i}}^{D}\right)$, whereas the agent's implied exposure to the output of agent's on other teams $\left(\beta_{i j}^{D}\right)$ is distorted upward, relative to the setting without public disclosure. 
Proposition X (Ex Ante Disclosure). Suppose teams have the same size $N_{I}$ and agents have uniform internal and external peer weights: $\mu_{i i}=0, \mu_{i \hat{i}}=\mu_{t}$ for $\hat{i} \in I \backslash i$ and $\mu_{i j}=\mu_{e}$ for $j \notin I$. Then the equilibrium contracts with ex ante disclosure have uniform internal and external loadings: $y_{i i}^{D}, y_{i \hat{i}}=y_{t}^{D}$ and $y_{i j}=y_{e}^{D}$ with exposures:

$$
\begin{gathered}
\beta_{i i}^{D}=\left(1+\alpha_{I}\right) y_{i i}^{*}-v y_{i i}^{*}, \\
\beta_{i \hat{i}}^{D}=-\frac{1}{n} \bar{\theta} \beta_{i i}^{D}-\left(1+\frac{1}{n} \bar{\theta}\right)\left(\frac{y_{i i}^{*}}{1-y_{i i}^{*}}\right) v, \text { and } \\
\beta_{i j}^{D}=-\frac{1}{n} \bar{\theta} \beta_{i i}^{D}+\left(\frac{n}{\bar{\theta}\left(N-N_{I}\right)}-1\right)\left(1+\frac{1}{n} \bar{\theta}\right)\left(\frac{y_{i i}^{*}}{1-y_{i i}^{*}}\right) v,
\end{gathered}
$$

where $v=\bar{\theta}\left(\frac{\delta^{2}}{1-\delta}\right)\left(\frac{N-N_{I}}{N-1}\right) N_{I} \mu_{e}^{2}\left(y_{i i}^{D}+\left(N_{I}-1\right) y_{t}^{D}+\left(N-2 N_{I}\right) y_{e}^{D}\right)>0$ and

$$
\alpha_{I}=\frac{\delta}{1-\delta}\left(1-\mu_{I}-N_{I}\left(N-N_{I}\right) \mu_{e} y_{e}^{D}\right)
$$

Without ex ante disclosure, (50) holds with $v=0$.

Proof: See Appendix. 


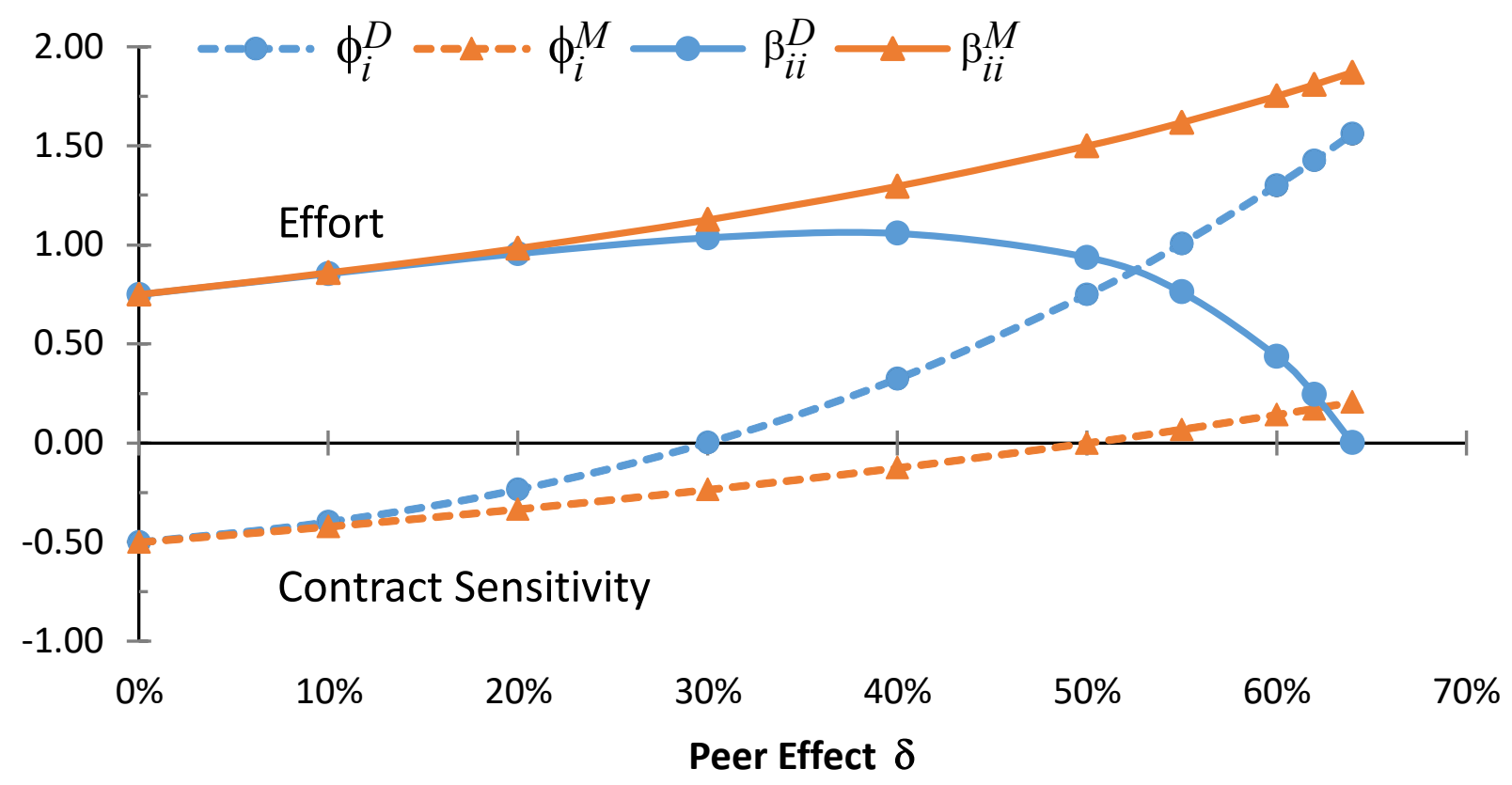

Figure 7: Effort and Contract Sensitivity with Ex Ante Disclosure

Ex ante disclosure increases contract sensitivity to external peers and decreases effort relative to case when contracts are not externally disclosed. (Parameters: $N=2, N_{I}=1, \bar{\theta}=50 \%, y_{i i}^{*}=75 \%$ ).

Figure 7 shows the effect of ex ante disclosure on both effort and contract sensitivity. Note that the effect of public disclosure increases with strength of peer effects $\delta$. Indeed, when $\delta$ sufficiently high, public disclosure is can cause effort to collapse well below the second-best level, in contrast the settings without disclosure where it is always above the second-best.

Thus, ex ante disclosure is likely to lead to even greater departures from "RPE" in observed contracts. And although public disclosure lowers equilibrium productivity, because effort is inefficiently high due to the rat race effect, this decrease in effort can raise profitability.

\section{Conclusion}

In this paper we have extended a standard moral hazard optimal contracting framework to a setting in which agents care about both their absolute wage, as well as how their wage compares to that of their peers. We allow for a general network of peer relationships, both within and across firms. Our results overturn standard predictions from contracting models. We find that rat race effects across teams raise equilibrium effort, and that compensation benchmarking offsets performance benchmarking, so that optimal contracts load more positively on peer output 
(including indirect peers) than the standard RPE model would imply. When peer effects are sufficiently strong, effort can exceed first best, while at the same time wages are driven primarily by aggregate (rather than individual) performance. Finally, principals can exploit asymmetric peer effects within their teams and raise profits, relative to when agents' preferences are devoid of peer effects, by inefficiently shifting effort to less visible agents.

We also considered the implications of different levels of disclosure and transparency. On one hand, if contract terms are not disclosed internally to teammates, a rat race effect emerges even within teams which raises team effort and output and lowers profits. On the other hand, external disclosure across teams is likely to increase the saliency of peer comparisons, exacerbating the rat race effect across teams and increasing wage levels and correlations.

In addition to these broad predictions, our model makes more nuanced predictions regarding the relationship between contract sensitivities and the details of peer relationships. In particular, we show that relative to RPE contracts, peer effects imply that contracts will be less sensitive to the agent's own output, and will have higher (more positive) sensitivity to peer output. The contract loading on peers will be highest for peers who are "closer" in the network, such as teammates or neighbors. With data on social networks becoming more readily available to researchers, one could envision merging such data with data on compensation to empirically evaluate these predictions.

To highlight these effects, we have kept all other aspects of the principal-agent model symmetric. In practice, we might expect the correlation between agents' output will also vary with some measure of distance. In that case the contract loadings will depend on relative distance between peers in the social network versus the production matrix.

In the multi-team context, we have also treated team and population size as exogenous. It would be interesting to explore the consequences of peer effects on industry structure when entry and exit is endogenous. For example, although we have assumed constant returns to scale in our model, larger teams are more efficient due to their ability to internalize rat race effects and thereby prevent wages from escalating. Conversely, rat race effects may be an important barrier to entry for smaller entrants to an industry. 


\section{Appendix}

Proof Of Lemma 1: When $\delta=0$, the contracting problem for agent $i$ is independent of the wage contracts of other agents, except to the extent that other agents' actions affect the expectation of the benchmark $q_{-i}$. Because $E\left[c_{i}\right]=\left(y_{i} \cdot 1\right) q_{0}+y_{i i} a_{i}+\sum_{j \in \mathcal{N}_{i}} y_{i j} a_{j}-\Psi\left(a_{i}\right)$, the agent will choose effort $a_{i}$ to maximize $y_{i i} a_{i}-\Psi\left(a_{i}\right)$, and thus $a_{i}=y_{i i} / k$. The principal will choose $y_{i 0}$ so that the agent's participation constraint binds; therefore $E\left[c_{i}\right]-\frac{1}{2} \lambda \operatorname{Var}\left(c_{i}\right)=c_{0}$. Because $c_{i} \equiv v_{i}-\psi\left(a_{i}\right)$,

$$
\overbrace{E\left[v_{i}\right]=E\left[w_{i}\right]}^{\delta=0}=c_{0}+\Psi\left(a_{i}\right)+\frac{1}{2} \lambda \operatorname{Var}\left(c_{i}\right) \text {. }
$$

The principal therefore chooses $y_{i j} / y_{i i}$ to minimize variance as in (12), so that the volatility of consumption is $y_{i i} \bar{\sigma}$. Then, because $a_{i}=y_{i i} / k$, the principal chooses $y_{i i}$ to trade off incentives for effort and the cost of risk-bearing in order to maximize

$$
\begin{aligned}
\pi_{i}=E\left[q_{i}-w_{i}\right] & =q_{0}+a_{i}-\Psi\left(a_{i}\right)-\frac{1}{2} \lambda \operatorname{Var}\left(c_{i}\right)-c_{0} \\
& =q_{0}+y_{i i} / k-\frac{1}{2} k\left(y_{i i} / k\right)^{2}-\frac{1}{2} \lambda y_{i i}^{2} \bar{\sigma}^{2}-c_{0},
\end{aligned}
$$

which implies (14) and (15). The principal's expected profit is $\pi_{i}=q_{0}+\frac{1}{2} a_{i}^{*}-c_{0}$. Finally, note that the constant term of the wage contract is set so that the participation constraint (51) binds; hence, because $E\left[q_{j}\right]=E\left[q_{i}\right]=q_{0}+a_{i}^{*}$ by symmetry,

$$
y_{i 0}^{*} q_{0}=c_{0}+\frac{1}{2} k\left(a_{i}^{*}\right)^{2}+\frac{1}{2} \lambda\left(y_{i i}^{*}\right)^{2} \bar{\sigma}^{2}-\sum_{j} y_{i j}^{*} E\left[q_{j}\right]=c_{0}+\frac{1}{2} a_{i}^{*}-k a_{i}^{*}(1-\bar{\theta})\left(q_{0}+a_{i}^{*}\right) .
$$

ProOf OF Proposition I: Note first that the participation constraint for each agent must bind, as otherwise the principal could cut the fixed component of the wage, increasing profits and (due to peer effects) relaxing the participation constraint of other agents. Therefore,

$$
\begin{aligned}
E\left[v_{i}\right] & =c_{0}+\psi\left(a_{i}\right)+\frac{1}{2} \lambda \operatorname{Var}\left(c_{i}\right) \\
& =c_{0}+\psi\left(\beta_{i i} / k\right)+\frac{1}{2} \lambda\left[(1-\rho) \sum_{j} \beta_{i j}^{2}+\rho\left(\sum_{j} \beta_{i j}\right)^{2}\right] \sigma_{\epsilon}^{2} \equiv C\left(\beta_{i}\right),
\end{aligned}
$$


where the function $C$ is the implied total cost borne by the agent given sensitivities $\beta$. Rewriting (53) in column vector form by stacking the equation for each agent $i$, and using the fact that $v=\Delta w$ and $\Delta$ is invertible (it has a strictly dominant diagonal), we have

$$
\Delta^{-1} E[v]=\Delta^{-1} E[\Delta w]=E[w]=\Delta^{-1} C(\beta),
$$

where we write $C(\beta)$ to be the column vector with row $i$ equal to $C\left(\beta_{i}\right)$. Next let $1 \in \mathcal{R}^{N \times 1}$ denote the column vector of ones. Then the principal's objective becomes

$$
\begin{aligned}
E\left[\sum_{i \in \mathcal{N}} q_{i}-w_{i}\right] & =1^{\prime} E[q-w]=1^{\prime} E[q]-1^{\prime} \Delta^{-1} C(\beta)=1^{\prime}(E[q]-C(\beta)) \\
& =E\left[\sum_{i \in \mathcal{N}} q_{i}-C\left(\beta_{i}\right)\right],
\end{aligned}
$$

where the penultimate step follows because $\mu$ is doubly stochastic ( $\mu 1=1$ from (1) and $\mu$ is symmetric implies $1^{\prime} \mu=1^{\prime}$ ) and therefore $\Delta$ and $\Delta^{-1}$ are doubly stochastic ( $\Delta$ is a convex combination of $I$ and $\mu$, which are both doubly stochastic, and $1=\Delta^{-1} \Delta 1=\Delta^{-1} 1$ ), and hence $1^{\prime} \Delta^{-1}=1^{\prime}$. Because $E\left[q_{i}\right]=c_{0}+a_{i}$ also depends only on $\beta_{i i}$, the solution $\beta^{*}$ to (55) does not depend on $\delta$, and so the solution matches the RPE case with $\delta=0$; i.e., $\beta^{*}=y^{*}$. The optimal contract $y^{S}$ then follows from $\beta^{*}=\Delta y^{S}$.

Proof of Corollary A: From (21), $w^{*}=y^{*} q=\Delta y^{S} q=\Delta w^{s}$ and given the definition of $\Delta$, we have

$$
(1-\delta) w^{*}=w^{S}-\delta \mu w^{S}=w^{S}-\delta w_{-i}^{S},
$$

which is equivalent to (22). Pre-multiplying by $1^{\prime}$, and using $1^{\prime} \mu=1^{\prime}$, shows that the aggregate wages and the wage benchmarks are all identical.

Proof of LemMa 2: For an $N$-row matrix, $M$, let $M_{I}$ be the matrix formed from rows $i \in I$, and $M_{J}$ be the matrix formed from rows $J=\mathcal{N} \backslash I$. Similarly, let $M_{I I}$ and $M_{I J}$ select the 
corresponding rows and columns (where we interpret column $0 \in I$ when relevant). Then, because $\beta=\Delta y$,

$$
\beta_{I}=\Delta_{I} y=\left[\begin{array}{ll}
\Delta_{I I} & \Delta_{I J}
\end{array}\right]\left[\begin{array}{l}
y_{I} \\
y_{J}
\end{array}\right]=\Delta_{I I} y_{I}+\Delta_{I J} y_{J}
$$

which we can rearrange as,

$$
\Delta_{I I} y_{I}=\beta_{I}-\Delta_{I J} y_{J}
$$

Because $\Delta_{I I}$ is invertible (it has a strictly dominant diagonal), we can solve for $y_{I}$ in terms of $\beta_{I}$ and $y_{J}$ as follows:

$$
y_{I}=\left(\Delta_{I I}\right)^{-1}\left[\beta_{I}-\Delta_{I J} y_{J}\right]
$$

Equation (56) shows that the principal for team $I$ can implement any set of exposures $\beta_{I}$ with an appropriate set of contracts $y_{I}$. Using the definitions $w=y q$ and $\beta q=\Delta y q=\Delta w=v$, together with the binding participation constraint (53), we have

$$
\begin{aligned}
E\left[w_{I}\right] & =E\left[y_{I} q\right]=\left(\Delta_{I I}\right)^{-1} E\left[\beta_{I} q-\Delta_{I J} y_{J} q\right]=\left(\Delta_{I I}\right)^{-1} E\left[v_{I}-\Delta_{I J} y_{J} q\right] \\
& =\left(\Delta_{I I}\right)^{-1}\left[C\left(\beta_{I}\right)-\Delta_{I J} E\left[w_{J}\right]\right]
\end{aligned}
$$

Because $\Delta_{I J} \leq 0$, (57) reflects the fact that an increase in the wage of agent $j \notin I$ raises the cost of compensating agent $i \in I$. Because $\Delta_{I I}$ has constant row (and column) sums equal to $\left(\frac{1-\mu_{I} \delta}{1-\delta}\right)$, the principal's expected profit $\pi_{I}$ is equal to

$$
\begin{aligned}
E\left[\sum_{i \in I} q_{i}-w_{i}\right] & =1^{\prime} E\left[q_{I}\right]-1^{\prime}\left(\Delta_{I I}\right)^{-1}\left[C\left(\beta_{I}\right)-\Delta_{I J} y_{J} E[q]\right] \\
& =1^{\prime} E\left[q_{I}\right]-\left(\frac{1-\delta}{1-\mu_{I} \delta}\right)\left[1^{\prime} C\left(\beta_{I}\right)-1^{\prime} \Delta_{I J} y_{J} E[q]\right] \\
& =\left(1^{\prime}+\left(\frac{1-\delta}{1-\mu_{I} \delta}\right) 1^{\prime} \Delta_{I J} y_{J I}\right) E\left[q_{I}\right]-\left(\frac{1-\delta}{1-\mu_{I} \delta}\right)\left[1^{\prime} C\left(\beta_{I}\right)-1^{\prime} \Delta_{I J} y_{J J} E\left[q_{J}\right]\right] \\
& =\left(\frac{1-\delta}{1-\mu_{I} \delta}\right)\left(\left(\left(\frac{1-\mu_{I} \delta}{1-\delta}\right) 1^{\prime}+1^{\prime} \Delta_{I J} y_{J I}\right) E\left[q_{I}\right]-1^{\prime} C\left(\beta_{I}\right)+1^{\prime} \Delta_{I J} y_{J J} E\left[q_{J}\right]\right)
\end{aligned}
$$


Finally, removing terms that do not depend on $y_{I}$, maximizing $\pi_{I}$ is equivalent to maximizing

$$
\begin{aligned}
& \sum_{i \in I}\left(\left(\frac{1-\mu_{I} \delta}{1-\delta}\right)+\sum_{\hat{i} j} \Delta_{\hat{i} j} y_{j i}\right) a_{i}-C\left(\beta_{i}\right) \\
& \quad=\sum_{i \in I}\left(1+\frac{\delta}{1-\delta}\left(1-\mu_{I}-\sum_{\hat{i} j} \mu_{\hat{i} j} y_{j i}\right)\right) a_{i}-C\left(\beta_{i}\right) \\
& \quad=\sum_{i \in I}\left(1+\alpha_{i}\right) a_{i}-C\left(\beta_{i}\right)
\end{aligned}
$$

Hence, principal $I$ will choose $\beta_{i}$ to maximize $\left(1+\alpha_{i}\right) a_{i}-C\left(\beta_{i}\right)$. From the incentive constraint $a_{i}=\beta_{i i} / k, \beta_{i i}$ maximizes

$$
\left(1+\alpha_{i}\right) \beta_{i i} / k-C\left(\beta_{i}\right)
$$

As in LEMMA 1, at the optimum $\beta_{i j}^{M}=-\frac{1}{n} \bar{\theta} \beta_{i i}^{M}$ to minimize variance, but the agent's sensitivity to his own output is distorted by the factor $\alpha_{i}$, with $\beta_{i i}^{M}=\left(1+\alpha_{i}\right) y_{i i}^{*}$. The optimal contract for team $I$ then follows from (56).

Proof of Proposition II: In a symmetric equilibrium, $\alpha_{i}=\alpha$, and Lemma 2 implies the principals choose sensitivities $\beta^{M}=(1+\alpha) y^{*}$. Because $\beta^{M}=\Delta y^{M}$,

$$
y^{M}=\Delta^{-1} \beta^{M}=\Delta^{-1}(1+\alpha) y^{*}=(1+\alpha) y^{S} .
$$

Conditions (25) and (26) imply that $\mu_{I}=1-\mu_{e}\left(N-N_{I}\right)$ and also that $\Delta_{i j}^{-1}=\lambda_{e}$ is constant for agents on different teams. Because $\Delta^{-1} \Delta=I,{ }^{26}$

$$
\lambda_{e}=\frac{\delta \mu_{e}}{1-\delta\left(1-N \mu_{e}\right)} \in\left[0, \frac{1}{N}\right)
$$

${ }^{26}$ We can write $\Delta=A-\frac{\delta}{1-\delta} \mu_{e} 11^{\prime}$ and $\Delta^{-1}=B+\lambda_{e} 11^{\prime}$ where both $A$ and $B$ are block diagonal with $A B=I$. Because $A 1=\Delta 1+\frac{\delta}{1-\delta} \mu_{e} 11^{\prime} 1=1+\frac{\delta}{1-\delta} \mu_{e} N 1$ and $A^{-1} 1=B 1=\Delta^{-1} 1-\lambda_{e} 11^{\prime} 1=1-\lambda_{e} N 1$, the value of $\lambda_{e}$ follows from the fact that we must have $1+\frac{\delta}{1-\delta} \mu_{e} N=\left(1-\lambda_{e} N\right)^{-1}$. 
Therefore, from $y^{S}=\Delta^{-1} y^{*}$,

$$
\frac{y_{e}^{S}}{y_{i i}^{*}}=\left(\lambda_{e}-\frac{1}{n} \bar{\theta}\left(1-\lambda_{e}\right)\right)=\frac{\delta \mu_{e}(1-\bar{\theta})-\frac{1}{n} \bar{\theta}(1-\delta)}{1-\delta+N \delta \mu_{e}} \in\left[-\frac{1}{n} \bar{\theta}, \lambda_{e}\right) .
$$

As an aside, note that $y_{e}^{S}>0$ iff

$$
\frac{\delta}{1-\delta}>\frac{\bar{\theta}}{n \mu_{e}(1-\bar{\theta})}
$$

Finally, from (24),

$$
\alpha=\frac{\delta}{1-\delta}\left(1-\mu_{I}-\sum_{\hat{i} \in I, j \notin I} \mu_{e} y_{e}^{M}\right)=\frac{\delta}{1-\delta}\left(1-\mu_{I}\right)\left(1-N_{I}(1+\alpha) y_{e}^{S}\right),
$$

and (28) follows by solving for $1+\alpha$. Because $N_{I} y_{e}^{S}<N_{I} / N<1$, we have $\alpha>0$. The expected wages and profit per agent follow because, by symmetry, $E\left[w_{i}\right]=E\left[v_{i}\right]=C_{i}$, and

$$
\begin{aligned}
\pi_{i}^{M}=E\left[q_{i}-w_{i}\right] & =q_{0}+a_{i}^{M}-\Psi\left(a_{i}^{M}\right)-\frac{1}{2} \lambda \operatorname{Var}\left(c_{i}\right)-c_{0} \\
& =q_{0}+(1+\alpha) a_{i}^{*}-\Psi\left((1+\alpha) a_{i}^{*}\right)-\frac{1}{2} \lambda(1+\alpha)^{2} y_{i i}^{* 2} \bar{\sigma}^{2}-c_{0} \\
& =\pi_{i}^{*}-\frac{y_{i i}^{*}}{2 k} \alpha^{2}=\pi_{i}^{*}-\frac{1}{2} a_{i}^{*} \alpha^{2} .
\end{aligned}
$$

Again, the constant component of the wage contract is set so that the agent's participation constraint binds:

$$
y_{i 0}^{M} q_{0}=c_{0}+\frac{1}{2} a_{i}^{*}(1+\alpha)^{2}-k a_{i}^{*}(1+\alpha)(1-\bar{\theta})\left(q_{0}+a_{i}^{*}(1+\alpha)\right) .
$$

Proof of Proposition III: From (28), as $\delta \rightarrow 1,1+\alpha \rightarrow \frac{1}{N_{I} y_{e}^{S}}$. For $\mu_{e}>0$ (equivalently, $N_{I}<N$ and $\mu_{I}<1$ ), from (61), $y_{e}^{S} \rightarrow \frac{1}{N}(1-\bar{\theta}) y_{i i}^{*}$. Combining these results and using $a^{*}=y_{i i}^{*} / k$ , we have 


$$
(1+\alpha) a^{*} \rightarrow \frac{N}{N_{I}(1-\bar{\theta})} \frac{1}{k},
$$

which implies that effort will exceed the first-best level $1 / k$ for $\delta$ sufficiently close to 1 .

To establish monotonicity in $\delta$, from (28) it suffices to show

$$
\frac{\partial}{\partial \delta}\left[\delta\left(\frac{1-\mu_{I}}{1-\delta \mu_{I}}\right)\left(1-N_{I} y_{e}^{S}\right)\right]>0 .
$$

Plugging in for $y_{e}^{S}$ from (61), taking the derivative, and simplifying we can show

$$
\begin{aligned}
& \overbrace{\left[\frac{(N-1)\left(1-\delta+\delta N_{\mu_{e}}\right)^{2}\left(1-\delta \mu_{I}\right)^{2}}{1-\mu_{I}}\right]}^{A>>} \frac{\partial}{\partial \delta}\left[\delta\left(\frac{1-\mu_{I}}{1-\delta \mu_{I}}\right)\left(1-N_{I} y_{e}^{S}\right)\right]= \\
& \underbrace{\left((1-\delta)\left(N-1+N_{I} y_{i i}^{*} \bar{\theta}\right)+(N-1) \delta\left(N-N_{I} y_{i i}^{*}(1-\bar{\theta})\right) \mu_{e}\right)\left(1-\delta+\delta N \mu_{e}\right)}_{B>0} \\
& -\underbrace{-N_{I} y_{i i}^{*} \delta(N-1+\bar{\theta}) \mu_{e}\left(1-\delta \mu_{I}\right)}_{C \geq 0}
\end{aligned}
$$

Because $0 \leq \delta, y_{i i}^{*}, \bar{\theta}, \mu_{I} \leq 1$, the terms $A, B$, and $C$ are positive; we thus need to show that $B-C \geq 0$. Note that a lower bound on $B$ is obtained by setting $\bar{\theta}=0$ and $y_{i i}^{*}=1$, and an upper bound for $C$ is obtained by setting $\bar{\theta}=y_{i i}^{*}=1$. These substitutions, together with the fact that $\left(N-N_{I}\right) \mu_{e}=1-\mu_{I}$, imply

$$
\begin{aligned}
B-C & \geq(N-1)\left(1-\delta+\delta\left(N-N_{I}\right) \mu_{e}\right)\left(1-\delta+\delta N \mu_{e}\right)-N_{I} \delta N \mu_{e}\left(1-\delta \mu_{I}\right) \\
& =(N-1)\left(1-\delta+\delta N \mu_{e}\right)\left(1-\delta \mu_{I}\right)-N N_{I} \delta \mu_{e}\left(1-\delta \mu_{I}\right)
\end{aligned}
$$

Dividing by $\left(1-\delta \mu_{I}\right)$ maintains the sign, and yields

$$
(N-1)(1-\delta)+N \delta\left(N-1-N_{I}\right) \mu_{e}>0,
$$

where the final inequality holds given $N_{I}<N-1$ with more than one principal. 
To show monotonicty in $N_{I}$, we show $1-\delta\left(\frac{1-\mu_{I}}{1-\delta \mu_{I}}\right)\left(1-N_{I} y_{e}^{S}\right)$ increases in $N_{I}$, where we of course need to keep in mind that $\mu_{I}$ and $y_{e}^{S}$ depend on $N_{I}$ as well. Furthermore, it suffices to focus on $N_{I} \leq \frac{1}{2} N$ since for $N_{I}=N, \alpha=0$. Taking the derivative with respect to $N_{I}$ and multiplying by

$$
\left(\delta \mu_{e}\right)^{-1}\left(1-\delta+\left(N-N_{I}\right) \delta \mu_{e}\right)^{2}(N-1)\left(1-\delta+\delta N \mu_{e}\right)>0
$$

and simplifying yields

$$
\begin{aligned}
& {\left[(N-1)(1-\delta)\left(1-\delta+\delta N \mu_{e}\right)\right]} \\
& \quad+y_{i i}^{*}\left(\left(N-2 N_{I}\right)(1-\delta)+\left(N-N_{I}\right)^{2} \delta \mu_{e}\right)\left((N-1) \delta \mu_{e}-\bar{\theta}\left(1-\delta+(N-1) \delta \mu_{e}\right)\right)
\end{aligned}
$$

Note that the square bracket is positive. Since $N \geq 2 N_{I},\left(\left(N-2 N_{I}\right)(1-\delta)+\left(N-N_{I}\right)^{2} \delta \mu_{e}\right) \geq 0$ and is decreasing in $N_{I}$. Hence, if $\left((N-1) \delta \mu_{e}-\theta\left(1-\delta+(N-1) \delta \mu_{e}\right)\right) \geq 0$, we are done. Otherwise, a lower bound for the whole expression is obtained by setting $y_{i i}^{*}=\bar{\theta}=N_{I}=1$, which after simplification yields

$$
\begin{gathered}
{\left[(N-1)(1-\delta)\left(1-\delta+\delta N \mu_{e}\right)\right]-(1-\delta)\left((N-2)(1-\delta)+(N-1)^{2} \delta \mu_{e}\right)} \\
=(N-1)(1-\delta)\left[\left(\frac{1}{N-1}\right)(1-\delta)+\delta \mu_{e}\right]>0
\end{gathered}
$$

Finally, we show $1-\delta\left(\frac{1-\mu_{I}}{1-\delta \mu_{I}}\right)\left(1-N_{I} y_{e}^{S}\right)$ decreases in $\mu_{e}$. Taking the derivative with respect to $\mu_{e}$, multiplying by

$$
\left[\left(N-N_{I}\right)(1-\delta) \delta\right]^{-1}(N-1)\left(1-\delta+\left(N-N_{I}\right) \delta \mu_{e}\right)^{2}\left(1-\delta+\delta N \mu_{e}\right)^{2}>0
$$

and simplifying yields 


$$
\begin{aligned}
& -(N-1)\left(1-\delta+\delta N \mu_{e}\right)^{2} \\
& +y_{i i}^{*} N_{I}\left[\begin{array}{c}
(N-1) \delta \mu_{e}\left(2(1-\delta)+\delta \mu_{e}\left(2 N-N_{I}\right)\right) \\
-\bar{\theta} \underbrace{\left(1-2 \delta+\delta^{2}+2(N-1)(1-\delta) \delta \mu_{e}+\left(N_{I}+(N-2) N\right) \delta^{2} \mu_{e}^{2}\right)}_{>0}
\end{array}\right]
\end{aligned}
$$

Because the first term is negative, if the square bracket term is negative as well we are done. We can maximize the above by setting $\bar{\theta}=0$ and $N_{I}=1$ inside [·], and $y_{i i}^{*}=1$ and $N_{I}=N / 2$ outside [.]. Substituting and simplifying yields

$$
\begin{gathered}
(N-1)\left[-\left(1-\delta+\delta N \mu_{e}\right)^{2}+\delta N \mu_{e}\left(1-\delta+\delta N \mu_{e}-\frac{1}{2} \delta \mu_{e}\right)\right] \\
<-(N-1)\left(1-\delta+\delta N \mu_{e}\right)(1-\delta)<0
\end{gathered}
$$

Proof OF Corollary B: The limiting case is immediate from (28) and $\left|y_{e}^{S}\right| \leq \frac{1}{n}$ from (61). Also from (28), $\alpha$ is increasing in $N$ iff $y_{e}^{S}$ is decreasing. As $N$ increases, $y_{e}^{S} \rightarrow 0$, and the highest order term in $N$ of $\frac{\partial}{\partial N} y_{e}^{S}$ is proportional to $N^{-2}\left(\bar{\theta}\left(1-\delta \mu_{I}\right)-\delta\left(1-\mu_{I}\right)\right)<0$. Hence $\alpha$ increases with $N$ for $N$ sufficiently large if $\bar{\theta}<\delta \frac{\left(1-\mu_{I}\right)}{1-\delta \mu_{I}}$ (it can also be shown if this holds as an equality by checking the $N^{-1}$-order term). Otherwise, the convergence is either decreasing or humpshaped.

Proof of Proposition IV: From Lemma 2, because $\mu_{I}+N_{J} \mu_{e}=1$ and, in equilibrium, $y_{j i}=\left(1+\alpha_{j}\right) y_{e}^{S}$,

$$
\begin{aligned}
\alpha_{i} & \equiv \frac{\delta}{1-\delta}\left(1-\mu_{I}-\sum_{i \in I, j \neq I} \mu_{i j} y_{j i}\right)=\frac{\delta}{1-\delta}\left(N_{J} \mu_{e}-N_{I} N_{J} \mu_{e}\left(1+\alpha_{j}\right) y_{e}^{S}\right) \\
& =\underbrace{\frac{\delta}{1-\delta}\left(N_{J} \mu_{e}-N_{I} N_{J} \mu_{e} y_{e}^{S}\right)}_{A_{j}}-\underbrace{\frac{\delta}{1-\delta} N_{I} N_{J} \mu_{e} y_{e}^{S}}_{B} \alpha_{j}
\end{aligned}
$$


and symmetrically for $\alpha_{j}$. Thus,

$$
\begin{aligned}
\alpha_{i}-\alpha_{j} & =A_{i}-A_{j}+B\left(\alpha_{i}-\alpha_{j}\right) \\
& =\frac{A_{i}-A_{j}}{1-B}=\frac{\delta \mu_{e}}{1-\delta} \frac{N_{J}-N_{I}}{1-B}>0,
\end{aligned}
$$

where the final inequality follows because $N_{J}>N_{I}$ (by assumption) and $B<1$ as we will show below. Next note that

$$
\begin{aligned}
\alpha_{i}+\alpha_{j} & =A_{i}+A_{j}-B\left(\alpha_{i}+\alpha_{j}\right) \\
& =\frac{A_{i}+A_{j}}{1+B}>0,
\end{aligned}
$$

where the last inequality follows because $A_{i}$ and $A_{j}$ are both positive (which follows from $y_{e}^{S} \leq 1 / N$ from (61)), and $B>-1$ as we show next. Together, (64) and (65) establish $\alpha_{i}>\left|\alpha_{j}\right|$, and the ranking of expected profits follows by the logic of (30).

Finally, we need to confirm $|B|<1$. From (61),

$$
\mu_{e} y_{e}^{S}=\mu_{e}\left(\frac{\delta \mu_{e}}{1-\delta\left(1-N \mu_{e}\right)}\left(1+\frac{1}{n} \bar{\theta}\right)-\frac{1}{n} \bar{\theta}\right) y_{i i}^{*}
$$

which is minimized by setting $\bar{\theta}=y_{i i}^{*}=1$ and $\mu_{e}=1 / N$; hence,

$$
\mu_{e} y_{e}^{S} \geq \frac{1}{N}\left(\frac{\delta}{N}\left(\frac{N}{N-1}\right)-\frac{1}{N}\right)=\frac{-(1-\delta)}{N(N-1)} .
$$

Therefore

$$
B=\frac{\delta}{1-\delta} N_{I} N_{J} \mu_{e} y_{e}^{S} \geq-\delta \frac{N_{I} N_{J}}{N(N-1)}>-1
$$

Next note that (66) is maximized by setting $\bar{\theta}=0, y_{i i}^{*}=1$, and $\mu_{e}=1 / N$; hence,

$$
\mu_{e} y_{e}^{S} \leq \frac{\delta}{N^{2}} .
$$

Therefore, 


$$
B=\frac{\delta}{1-\delta} N_{I} N_{J} \mu_{e} y_{e}^{S} \leq \frac{\delta^{2}}{1-\delta} \frac{N_{I} N_{J}}{N^{2}}<\frac{\frac{1}{4} \delta^{2}}{1-\delta}<1
$$

where the last inequality holds for $\delta<2(\sqrt{2}-1) \approx 0.828$.

Proof Of Proposition V: We can write $y^{*}$ as

$$
y^{*}=y_{i i}^{*}\left[\left(1+\frac{1}{n} \bar{\theta}\right) I-\frac{1}{n} \bar{\theta} 11^{\prime}\right]
$$

and because $\Delta 1=1 \Rightarrow \Delta^{-1} 1=1$, we have

$$
y^{S}=\Delta^{-1} y^{*}=y_{i i}^{*}\left[\left(1+\frac{1}{n} \bar{\theta}\right) \Delta^{-1}-\frac{1}{n} \bar{\theta} 11^{\prime}\right] .
$$

Equation (68) implies $y_{i j}^{S} / y_{i i}^{*} \in\left[-\frac{1}{n} \bar{\theta}, 1\right]$, and $y_{i i}^{S} \geq y_{i j}^{S}$ follows from $\Delta_{i i}^{-1} \geq \Delta_{i j}^{-1}$, which we establish below. For the overall peer sensitivity, $y^{S} 1=\Delta^{-1} y^{*} 1=y_{i i}^{*}\left[\left(1+\frac{1}{n} \bar{\theta}\right)-\frac{n+1}{n} \bar{\theta}\right] 1$ implies

$$
\begin{aligned}
\phi_{i}^{S} & \equiv \frac{\sum_{j \in \mathcal{N}_{i}} y_{i j}^{S}}{y_{i i}^{S}}=\frac{\sum_{j} y_{i j}^{S}}{y_{i i}^{S}}-1=\frac{\left(y^{s} 1\right)_{i}}{\left(\Delta^{-1} y^{*}\right)_{i i}}-1=\frac{\left(1+\frac{1}{n} \bar{\theta}\right)-\frac{n+1}{n} \bar{\theta}}{\left(1+\frac{1}{n} \bar{\theta}\right) \Delta_{i i}^{-1}-\frac{1}{n} \bar{\theta}}-1 \\
& =\frac{1-\bar{\theta}}{\left(1+\frac{1}{n} \bar{\theta}\right) \Delta_{i i}^{-1}-\frac{1}{n} \bar{\theta}}-1
\end{aligned}
$$

Thus the behavior of $\phi_{i}^{S}$ is determined by $\Delta_{i i}^{-i}$. Specifically, $-\bar{\theta}<\phi_{i}^{S}<n$ is equivalent to $1>\Delta_{i i}^{-1}>1 / N$. To bound $\Delta_{i i}^{-1}$ from above, note that $\Delta \equiv \frac{1}{1-\delta}[I-\delta \mu]$ implies

$$
\Delta^{-1}=(1-\delta) I+\delta \mu \Delta^{-1}=(1-\delta)\left[I+\delta \mu+\delta^{2} \mu^{2}+\ldots\right],
$$

and therefore $\Delta^{-1}$ is a symmetric, strictly positive (since $\mu$ is irreducible), stochastic matrix. Because $\mu_{i i}<1$ and $\mu_{i i}^{t} \leq 1$ for all $t$, for $\delta \in(0,1)$ we have

$$
\Delta_{i i}^{-1} \leq(1-\delta)\left(1+\delta \mu_{i i}+\delta^{2}+\delta^{3}+\ldots\right)=1-(1-\delta)\left(1-\mu_{i i}\right) \delta<1 .
$$

To bound $\Delta_{i i}^{-1}$ from below, we show next that the diagonal elements of $\Delta^{-1}$ are larger than the off-diagonal elements. Suppose instead that there exists $\hat{j} \neq i$ such that $\Delta_{i j}^{-1}=\max _{j} \Delta_{i j}^{-1}$. But 
then $\delta\left(\Delta^{-1} \mu\right)_{\hat{i} j}=\delta \sum_{k} \Delta_{i k}^{-1} \mu_{k \hat{j}} \leq \delta \max _{k} \Delta_{i k}^{-1}<\Delta_{i \hat{j}}^{-1}$, contradicting $\Delta^{-1}=(1-\delta) I+\delta \Delta^{-1} \mu$. Hence $\Delta_{i i}^{-1}=\max _{j} \Delta_{i j}^{-1} \geq 1 / N$

In the limit when $\delta=1,(70)$ implies $\Delta^{-1}=\mu \Delta^{-1}=\mu^{t} \Delta^{-1}$. Because $\mu$ is ergodic and doubly stochastic, $\mu_{i j}^{t} \rightarrow 1 / N$ as $t \rightarrow \infty$, and therefore $\Delta_{i j}^{-1} \rightarrow 1 / N$. Therefore, $y_{i j}^{S} \rightarrow y_{i i}^{*}(1-\bar{\theta}) / N$.

Finally, from (70),

$$
\begin{aligned}
\frac{\partial}{\partial \delta} \Delta^{-1} & =\frac{\partial}{\partial \delta}\left[(1-\delta) I+\delta \mu \Delta^{-1}\right]=-\left(I-\mu \Delta^{-1}\right)+\delta \mu \frac{\partial}{\partial \delta} \Delta^{-1} \\
& =-(I-\delta \mu)^{-1}\left(I-\mu \Delta^{-1}\right) \\
= & -\underbrace{\left(\frac{1}{1-\delta} \Delta^{-1}\right)}_{\substack{\text { from } \\
\Delta=\frac{1}{1-\delta}[I-\delta \mu]}} \underbrace{\left(\frac{1}{\delta}\left(I-\Delta^{-1}\right)\right)}_{\text {from }}=\frac{-1}{\delta(1-\delta) I+\delta \mu \Delta^{-1}}\left(\Delta^{-1}-\Delta^{-1} \Delta^{-1}\right)
\end{aligned}
$$

Because $\Delta_{i i}^{-1}=\max _{j} \Delta_{i j}^{-1}>\min _{j} \Delta_{i j}^{-1}$ for $\delta \in(0,1)$, we have

$$
\begin{aligned}
\left(\Delta^{-1}-\Delta^{-1} \Delta^{-1}\right)_{i i} & =\Delta_{i i}^{-1}-\sum_{j}\left(\Delta_{i j}^{-1}\right)^{2} \\
& >\Delta_{i i}^{-1}-\left(\max _{j} \Delta_{i j}^{-1}\right) \underbrace{\sum_{j} \Delta_{i j}^{-1}}_{=1} \\
& =\Delta_{i i}^{-1}-\Delta_{i i}^{-1}=0
\end{aligned}
$$

and thus $\frac{\partial}{\partial \delta} \Delta_{i i}^{-1}<0$.

Proof of Proposition VI: With uniform internal and external weights, the symmetric matrix $\mu$ can be diagonalized with three distinct eigenvalues

$$
\left\{e_{1}, e_{a}, e_{b}\right\}=\{1, \overbrace{\mu_{I}-N_{I} \mu}^{\text {multiplicity } \frac{N}{N_{I}}-1}, \overbrace{-\mu_{t}=-\mu_{I} / n_{I}}^{\text {multiplicity } \frac{N}{N_{I}} n_{I}} \text {. }
$$

It is straightforward to show that $\Delta^{-1}$ has the same structure with eigenvalues

$$
\left\{1, a=\frac{1-\delta}{1-\delta e_{a}}, b=\frac{1-\delta}{1-\delta e_{b}}\right\}
$$

and thus has corresponding external, internal, and diagonal elements: 


$$
\Delta_{e}^{-1}=\frac{1-a}{N}, \Delta_{t}^{-1}=\Delta_{e}^{-1}+\frac{a-b}{N_{I}}, \Delta_{i i}^{-1}=\Delta_{t}^{-1}+b
$$

Equilibrium contracts are given by $y^{M}=(1+\alpha) y^{S}=(1+\alpha) \Delta^{-1} y_{i i}^{*}$, with external, internal, and own exposures

$$
\frac{y_{x}^{S}}{y_{i i}^{*}}=\left(1+\frac{1}{n} \bar{\theta}\right) \Delta_{x}^{-1}-\frac{1}{n} \bar{\theta}
$$

Because $\Delta_{e}^{-1}=\frac{1-a}{N}=\frac{\delta\left(1-e_{a}\right)}{N\left(1-\delta e_{a}\right)}=\frac{\delta \mu_{e}}{1-\delta+N \delta \mu_{e}}$, we have

$$
y_{e}^{S}=\frac{\delta \mu_{e}(1-\bar{\theta})-\frac{1}{n} \bar{\theta}(1-\delta)}{1-\delta+N \delta \mu_{e}} y_{i i}^{*},
$$

which is increasing in $\mu_{e}$ and implies (37). Because the rat race factor $\alpha$ is also increasing in $\mu_{e}$ from Proposition III, $y_{e}^{M}$ is also increasing in $\mu_{e}$.

Note that

$$
\frac{\partial}{\partial \mu_{e}} \Delta_{i i}^{-1}=\left(\frac{1}{N_{I}}-\frac{1}{N}\right) a^{\prime}+\left(1-\frac{1}{N_{I}}\right) b^{\prime}=\left(\frac{1}{N_{I}}-\frac{1}{N}\right) \frac{\delta(1-\delta)}{\left(1-\delta e_{a}\right)^{2}} e_{a}{ }^{\prime}+\left(\frac{n_{I}}{N_{I}}\right) \frac{\delta(1-\delta)}{\left(1-\delta e_{b}\right)^{2}} e_{b}{ }^{\prime},
$$

and because $e_{b} \leq e_{a} \leq 1, e_{a}{ }^{\prime}=-N$, and $e_{b}{ }^{\prime}=\left(N-N_{I}\right) / n_{I}$,

$$
\frac{\partial}{\partial \mu_{e}} \Delta_{i i}^{-1}=\delta(1-\delta)\left(\frac{N}{N_{I}}-1\right)\left(\frac{1}{\left(1-\delta e_{b}\right)^{2}}-\frac{1}{\left(1-\delta e_{a}\right)^{2}}\right) \leq 0,
$$

with equality if and only if $\mu_{e}=1 / n$. Hence (33) implies $\phi_{i}$ is increasing in $\mu_{e}$.

When $\mu_{e}=0$, then $\mu_{I}=e_{a}=a=1$ and so $\Delta_{e}^{-1}=0$ and $y_{e}=-\frac{1}{n} \bar{\theta}$. Because $\alpha=0$ and $\Delta_{t}^{-1}=\frac{1-b}{N_{I}}=\frac{\delta\left(1-e_{b}\right)}{N_{I}\left(1-\delta e_{b}\right)}=\frac{\delta\left(1+\frac{1}{n_{I}}\right)}{N_{I}\left(1+\frac{1}{n_{I}} \delta\right)}=\frac{\delta}{n_{I}+\delta}$, the optimal contract has team weights

$$
y_{t}^{M}=y_{t}^{S}=\left(\frac{\delta-\frac{n_{I}}{n} \bar{\theta}}{n_{I}+\delta}\right) y_{i i}^{*}
$$


Proof OF Corollary C: With equal weights,

$$
\Delta=\frac{1+\frac{1}{n} \delta}{1-\delta} I-\frac{\frac{1}{n} \delta}{1-\delta} 11^{\prime}, \Delta^{-1}=\frac{1-\delta}{1+\frac{1}{n} \delta} I+\frac{\frac{1}{n} \delta}{1+\frac{1}{n} \delta} 11^{\prime}, \text { and } y^{*}=y_{i i}^{*}\left[\left(1+\frac{1}{n} \bar{\theta}\right) I-\frac{1}{n} \bar{\theta} 11^{\prime}\right]
$$

and the result then follows from (21). The rat race factor is immediate from (38) and (28).

Proof of Corollary D: From $(70), \Delta^{-1}=(1-\delta) I+\delta \mu \Delta^{-1}$ implies $\Delta_{i i}^{-1} \in\left[1-\delta, 1-\delta+\delta\|\mu\|_{\max }\right]$ . Then from (33),

$$
\phi_{i}^{S}=\frac{1-\bar{\theta}}{\left(1+\frac{1}{n} \bar{\theta}\right) \Delta_{i i}^{-1}-\frac{1}{n} \bar{\theta}}-1 \rightarrow \frac{1-\bar{\theta}}{1-\delta}-1
$$

and the result follows.

Proof of Corollary E: Let $d(i, j)$ be the distance between $i$ and $j$ on the circle. Then we can check that

$$
\Delta_{i j}^{-1}=\sqrt{\frac{1-\delta}{1+\delta}}\left(\frac{z^{d(i, j)}+z^{N-d(i, j)}}{1-z^{N}}\right) \text { where } z=\frac{1}{\delta}-\sqrt{\frac{1}{\delta^{2}}-1} \in(0,1) .
$$

Therefore, $\Delta_{i j}^{-1}>0$ is decreasing in $d(i, j)$, and thus $y_{i j}>-\frac{1}{n} \bar{\theta} y_{i i}$ and decreasing in $d(i, j)$. In addition,

$$
\Delta_{i i}^{-1}=\sqrt{\frac{1-\delta}{1+\delta}}\left(\frac{1+z^{N}}{1-z^{N}}\right) \rightarrow \sqrt{\frac{1-\delta}{1+\delta}} \text { as } N \rightarrow \infty
$$

and so from (33) the result follows because $\phi_{i}=\frac{1-\bar{\theta}}{\left(1+\frac{1}{n} \bar{\theta}\right) \Delta_{i i}^{-1}-\frac{1}{n} \bar{\theta}}-1 \rightarrow \frac{1-\bar{\theta}}{\Delta_{i i}^{-1}}-1$.

Proof of Corollary F: From (70), 


$$
\begin{aligned}
\frac{\partial}{\partial \lambda} \Delta_{i i}^{-1} & =\frac{\partial}{\partial \lambda}\left[(1-\delta) I+\delta \mu(\lambda) \Delta^{-1}\right]_{i i}=\left[\delta \mu^{\prime}(\lambda) \Delta^{-1}\right]_{i i} \\
& =\delta \sum_{j} \mu_{i j}^{\prime}(\lambda) \Delta_{j i}^{-1} \\
& =\delta \sum_{j} \mu_{i j}^{\prime}(\lambda)\left(\Delta_{j i}^{-1}-\Delta_{i i}^{-1}\right) \leftarrow \text { because } \mu^{\prime}(\lambda) 1=0 \\
& >0 \leftarrow \text { because } \Delta_{j i}^{-1} \leq \Delta_{i i}^{-1} .
\end{aligned}
$$

Hence, $\phi_{i}{ }^{\prime}(\lambda)<0$ from (33).

Proof of Proposition VII: Note first that $1^{\prime} \Delta^{-1}=1^{\prime}$ implies $1^{\prime} \Delta=1^{\prime} \Delta^{-1} \Delta=1^{\prime}$, and then because $\mu=\frac{1}{\delta} I-\frac{1-\delta}{\delta} \Delta$, we have $1^{\prime} \mu=\frac{1}{\delta} 1^{\prime}-\frac{1-\delta}{\delta} 1^{\prime} \Delta=1^{\prime}$. Therefore, $1^{\prime} \mu \equiv \bar{\mu} \neq 1^{\prime}$ implies $\omega^{\prime} \equiv 1^{\prime} \Delta^{-1} \neq 1^{\prime}$ . Moreover, because $\omega^{\prime} 1=1^{\prime} \Delta^{-1} 1=1^{\prime} 1=N$, by Jensen's inequality we have $\sum_{j} \omega_{j}^{-1}>N$.

Next, recall from (54) that the principal maximizes

$$
E\left[\sum_{i} q_{i}-w_{i}\right]=1^{\prime} E[q-w]=1^{\prime} E[q]-1^{\prime} \Delta^{-1} C(\beta)=E\left[\sum_{i} q_{i}-\omega_{i} C\left(\beta_{i}\right)\right],
$$

from which the optimal effort level follows. The principal's total expected payoff is

$$
N\left(q_{0}-c_{0}\right)+\frac{1}{2} a_{i}^{*}\left(\sum_{j} \omega_{j}^{-1}\right)>N\left(q_{0}-c_{0}+\frac{1}{2} a_{i}^{*}\right)=N \pi_{i}^{*}
$$

which improves upon the RPE payoff.

Proof of Proposition VIII: In this setting, we can represent $\Delta$ in block format as $\Delta=\left[\begin{array}{cc}I_{J} & 0_{J, N-J} \\ \Delta_{N-J, J} & \Delta_{N-J, N-J}\end{array}\right]$ with

$$
\Delta_{N-J, J}=-\frac{1}{n} \frac{\delta}{1-\delta} 1_{N-J} 1_{J}^{\prime}, \text { and } \Delta_{N-J, N-J}=\frac{1+\frac{1}{n} \delta}{1-\delta} I_{N-J, N-J}-\frac{1}{n} \frac{\delta}{(1-\delta)} 1_{N-J} 1_{N-J}^{\prime}
$$

Computing the inverse yields $\Delta^{-1}=\left[\begin{array}{cc}I_{J} & 0_{J, N-J} \\ \Delta_{N-J, J}^{-1} & \Delta_{N-J, N-J}^{-1}\end{array}\right]$ with

$$
\Delta_{N-J, J}^{-1}=\frac{1}{n} \frac{\delta}{1-(1-J / n) \delta} 1_{N-J} 1_{J}^{\prime}, \text { and }
$$




$$
\Delta_{N-J, N-J}^{-1}=\frac{1-\delta}{1+\frac{1}{n} \delta} I_{N-J, N-J}+\frac{1}{n} \frac{\delta}{\left(1+\frac{1}{n} \delta\right)} \frac{(1-\delta)}{(1-(1-J / n) \delta)} 1_{N-J} 1_{N-J}^{\prime} .
$$

Finally, by direct calculation, the effective cost for each agent type is

$$
\begin{aligned}
& \omega_{j}=1+\frac{(n+1-J) \delta}{n(1-\delta)+J \delta}=\frac{n+\delta}{n(1-\delta)+J \delta} \text { and } \\
& \omega_{m}=\frac{1-\delta}{1+\frac{1}{n} \delta}+\frac{(n+1-J) \delta}{\left(1+\frac{1}{n} \delta\right)} \frac{(1-\delta)}{n(1-\delta)+J \delta}=\frac{n(1-\delta)}{n(1-\delta)+J \delta}=\frac{1-\delta}{1+\frac{1}{n} \delta} \omega_{j}<\omega_{j} .
\end{aligned}
$$

Letting $\psi=\frac{1+\frac{1}{n} \delta}{1-\delta}>1$, from Proposition VII we have $\frac{a_{m}^{A}}{a_{j}^{A}}=\frac{\omega_{m}^{-1}}{\omega_{j}^{-1}} \equiv \psi$, and

$$
\begin{aligned}
\frac{1}{N} \sum_{j} \omega_{j}^{-1} & =\frac{1}{N}\left(J \omega_{j}^{-1}+(N-J) \omega_{m}^{-1}\right)=\frac{1}{N} \omega_{j}^{-1}(J+(N-J) \psi) \\
& =\left(\frac{n(1-\delta)+J \delta}{n+\delta}\right)\left(\frac{J}{N}+\left(1-\frac{J}{N}\right) \psi\right) \\
& =\left(\psi^{-1}+\frac{J}{N}\left(1-\psi^{-1}\right)\right)\left(\psi+\frac{J}{N}(1-\psi)\right) \\
& =1+\left(\psi+\psi^{-1}-2\right) \frac{J}{N}\left(1-\frac{J}{N}\right) .
\end{aligned}
$$

ProOf OF LemMa 3: Following the proof of LemMa 2, holding the contracts and actions of all other agents $j \in J=N_{i}$ fixed,

$$
\begin{aligned}
E\left[q_{i}-w_{i}\right] & =E\left[q_{i}\right]-\left(\Delta_{i i}\right)^{-1}\left[C\left(\beta_{i}\right)-\Delta_{i J} y_{J i} E\left[q_{i}\right]-\Delta_{i J} y_{J J} E\left[q_{J}\right]\right] \\
& =E\left[q_{i}\right]-\left(\frac{1-\delta}{1-\delta \mu_{i i}}\right)\left[C\left(\beta_{i}\right)-\Delta_{i J} y_{J i} E\left[q_{i}\right]-\Delta_{i J} y_{J J} E\left[q_{J}\right]\right] \\
& =\left(1+\left(\frac{1-\delta}{1-\delta \mu_{i i}}\right) \Delta_{i J} y_{J i}\right) E\left[q_{i}\right]-\left(\frac{1-\delta}{1-\delta \mu_{i i}}\right) C\left(\beta_{i}\right)+\left(\frac{1-\delta}{1-\delta \mu_{i i}}\right) \Delta_{i J} y_{J J} E\left[q_{J}\right] \\
& =\left(1-\frac{\delta}{1-\delta \mu_{i i}} \mu_{i J} y_{J i}\right) E\left[q_{i}\right]-\left(\frac{1-\delta}{1-\delta \mu_{i i}}\right) C\left(\beta_{i}\right)+\left(\frac{1-\delta}{1-\delta \mu_{i i}}\right) \Delta_{i J} y_{J J} E\left[q_{J}\right]
\end{aligned}
$$


In addition, by changing $E\left[q_{i}\right]$, the principal also changes the expected wage to agent $\hat{i}$ by $y_{\hat{i}}$. Therefore, letting $\hat{I}=I_{i}$, the principal will choose $\beta_{i}$ to maximize

$$
\left(1-\frac{\delta}{1-\delta \mu_{i i}} \mu_{i J} y_{J i}-1^{\prime} y_{\hat{i}}\right) E\left[q_{i}\right]-\left(\frac{1-\delta}{1-\delta \mu_{i i}}\right) C\left(\beta_{i}\right)=\left(\frac{1-\delta}{1-\delta \mu_{i i}}\right)\left[\left(1+\alpha_{i}^{R}\right) E\left[q_{i}\right]-C\left(\beta_{i}\right)\right]
$$

for

$$
1+\alpha_{i}^{R} \equiv\left(\frac{1-\delta \mu_{i i}}{1-\delta}\right)\left(1-\frac{\delta}{1-\delta \mu_{i i}} \sum_{j \neq i} \mu_{i j} y_{j i}-\sum_{i \in I_{i}} y_{\hat{i}}\right)
$$

which is solved by $\beta_{i}^{R}=\left(1+\alpha_{i}^{R}\right) y_{i}^{*}$.

Proof Of Proposition IX: Using (44) with symmetric $\alpha^{R}$,

$$
\begin{aligned}
1+\alpha^{R} & \equiv\left(\frac{1-\delta \mu_{i i}}{1-\delta}\right)\left(1-\frac{\delta}{1-\delta \mu_{i i}} \sum_{j \neq i} \mu_{i j} y_{j i}-\sum_{i \in I_{i}} y_{\hat{i} i}\right) \\
& =\frac{1}{1-\delta}\left(1-\delta \sum_{j \neq i} \mu_{i j} y_{j i}+y_{i i}-\sum_{i \in I} y_{\hat{i i t}}\right)=\frac{1}{1-\delta}+\Delta_{i \cdot} y_{i i}-\frac{1}{1-\delta} 1^{\prime} y_{l i} \\
& =\frac{1}{1-\delta}+\left(1+\alpha^{R}\right)\left(\left(\Delta \Delta^{-1} y^{*}\right)_{i i}-\frac{1}{1-\delta} \sum_{i \in I} y_{\hat{i} i}^{S}\right) \leftarrow \operatorname{using} y=\left(1+\alpha^{R}\right) y^{S} \text { and } y^{S}=\Delta^{-1} y^{*} \\
& =\frac{1}{1-\delta}+\left(1+\alpha^{R}\right)\left(y_{i i}^{*}-\frac{1}{1-\delta} \sum_{i \in I} y_{\hat{i}}^{S}\right) \\
& =\frac{1}{(1-\delta)\left(1-y_{i i}^{*}\right)+\sum_{i \in I} y_{\hat{i}}^{S} \leftarrow \operatorname{moving}\left(1+\alpha^{R}\right) \text { to the left-hand side }} \\
& =\frac{1}{(1-\delta)\left(1-y_{i i}^{*}\right)+\left(y_{i i}^{*}(1-\bar{\theta})-\left(N-N_{I}\right) y_{e}^{S}\right)}
\end{aligned}
$$

where the final step follows because

$$
\sum_{i \in I} y_{i i}^{S}+\left(N-N_{I}\right) y_{e}^{S}=1^{\prime} y^{S}=1^{\prime} \Delta^{-1} y^{*}=1^{\prime} y^{*}=y^{*} 1=y_{i i}^{*}(1-\bar{\theta})
$$

To show that $\alpha^{R}>\alpha$, note from (60) and (61) that 


$$
\lambda_{e}=\frac{\delta \mu_{e}}{1-\delta\left(1-N \mu_{e}\right)}=\frac{\delta\left(\frac{1-\mu_{I}}{N-N_{I}}\right)}{1-\delta+\delta N\left(\frac{1-\mu_{I}}{N-N_{I}}\right)}=\frac{\delta\left(1-\mu_{I}\right)}{N\left(1-\delta \mu_{I}\right)-N_{I}(1-\delta)},
$$

and

$$
y_{e}^{S}=\frac{1}{N-1}\left(\lambda_{e}(N-1+\bar{\theta})-\bar{\theta}\right) y_{i i}^{*}=\frac{1}{N-1}\left(\frac{\delta\left(1-\mu_{I}\right)(N-1+\bar{\theta})}{N\left(1-\delta \mu_{I}\right)-N_{I}(1-\delta)}-\bar{\theta}\right) y_{i i}^{*} .
$$

Therefore,

$$
\begin{aligned}
& \overbrace{1-\delta\left(\frac{1-\mu_{I}}{1-\delta \mu_{I}}\right)\left(1-N_{I} y_{e}^{S}\right)}^{\frac{1}{1+\alpha}}-\overbrace{\left(1-\delta+(\delta-\bar{\theta}) y_{i i}^{*}-\left(N-N_{I}\right) y_{e}^{S}\right)}^{\frac{1}{1+\alpha^{R}}} \\
& \quad=1-\delta\left(\frac{1-\mu_{I}}{1-\delta \mu_{I}}\right)-(1-\delta)-(\delta-\bar{\theta}) y_{i i}^{*}+\left(\delta\left(\frac{1-\mu_{I}}{1-\delta \mu_{I}}\right)-1\right) N_{I} y_{e}^{S}+N y_{e}^{S} \\
& =\frac{\delta(1-\delta) \mu_{I}}{1-\delta \mu_{I}}-(\delta-\bar{\theta}) y_{i i}^{*}+\left(\frac{N\left(1-\delta \mu_{I}\right)-N_{I}(1-\delta)}{1-\delta \mu_{I}}\right) y_{e}^{S} \\
& =\frac{(1-\delta) \mu_{I}}{1-\delta \mu_{I}}\left(\delta-\left(\delta-\frac{\left(N_{I}-1\right)}{(N-1) \mu_{I}}\right) y_{i i}^{*}\right) \\
& \geq 0,
\end{aligned}
$$

and the inequality is strict as long as $N_{I}>1$ so that $\mu_{I}>0$. The formula for $\alpha^{R}$ with equal weights on all agents follows from (38).

Proof of Corollary G: The rat race results follow from Proposition III. The effects on $y_{e}$ follow because, from (61),

$$
y_{e}^{S}=\frac{\delta \mu_{e}(1-\bar{\theta})-\frac{1}{n} \bar{\theta}(1-\delta)}{1-\delta+N \delta \mu_{e}} y_{i i}^{*},
$$

which is monotone in both $\delta$ and $\mu_{e}$. The final bullet follows from Proposition $\mathbf{V}$ and Proposition VI, and the fact that from (72) and (73), 


$$
\operatorname{sgn} \frac{\partial}{\partial \mu_{e}}\left(y_{t}^{S}-y_{e}^{S}\right)=\operatorname{sgn}\left(a^{\prime}-b^{\prime}\right)<0
$$

Proof of Proposition X: Following the steps of the proof of Lemma 2 up to (58), but now retaining the terms that depend on $q_{J}$, we see that maximizing $\pi_{I}$ is equivalent to maximizing

$$
\begin{aligned}
& \left(\sum_{i \in I}\left(\left(\frac{1-\mu_{I} \delta}{1-\delta}\right)+\sum_{\hat{i j}} \Delta_{\hat{i} j} y_{j i}\right) E\left[q_{i}\right]-C\left(\beta_{i}\right)\right)+1^{\prime} \Delta_{I J} y_{J J} E\left[q_{J}\right] \\
& \quad=\left(\sum_{i \in I}\left(1+\frac{\delta}{1-\delta}\left(1-\mu_{I}-\sum_{\hat{i} j} \mu_{\hat{i} j} y_{j i}\right)\right) E\left[q_{i}\right]-C\left(\beta_{i}\right)\right)-\left(\frac{\delta}{1-\delta}\right) \sum_{j \in J}\left(\sum_{\hat{i} k} \mu_{\hat{i} k} y_{k j}\right) E\left[q_{j}\right] \\
& \quad=\left(\sum_{i \in I}\left(1+\frac{\delta}{1-\delta}\left(1-\mu_{I}-N_{I}\left(N-N_{I}\right) \mu_{e} y_{e}\right)\right) E\left[q_{i}\right]-C\left(\beta_{i}\right)\right)-\left(\frac{\delta N_{I} \bar{y}}{1-\delta}\right) \sum_{j \in J} E\left[q_{j}\right]
\end{aligned}
$$

where $\bar{y}=\mu_{e}\left(y_{j j}+\left(N_{I}-1\right) y_{t}+\left(N-2 N_{I}\right) y_{e}\right)$. Recall that $a_{i}=\beta_{i i} / k$, and hence we can equivalently maximize

$$
\left(\sum_{i \in I}\left(1+\alpha_{I}\right) \beta_{i i}-k C\left(\beta_{i}\right)\right)-\gamma_{I} \sum_{j \in J} \beta_{j j}
$$

where $\alpha_{I}=\frac{\delta}{1-\delta}\left(1-\mu_{I}-N_{I}\left(N-N_{I}\right) \mu_{e} y_{e}\right)$ and $\gamma_{I}=\frac{\delta}{1-\delta} N_{I} \bar{y}$.

Recall that $\beta_{i j} \equiv \frac{\partial c_{i}}{\partial q_{j}}=\frac{y_{i j}-\delta \sum_{k} \mu_{i k} y_{k j}}{1-\delta}$ and

$$
C\left(\beta_{i}\right) \equiv c_{0}+\psi\left(\beta_{i i} / k\right)+\frac{1}{2} \lambda\left[(1-\rho) \sum_{j} \beta_{i j}^{2}+\rho\left(\sum_{j} \beta_{i j}\right)^{2}\right] \sigma_{\epsilon}^{2} .
$$

Taking the first-order condition of (79) with respect to $\beta_{i i}$ we have:

$$
1+\alpha_{I}=k \frac{\partial}{\partial \beta_{i i}} C\left(\beta_{i}\right)=\beta_{i i}+k \lambda \sigma_{\epsilon}^{2}\left[(1-\rho) \beta_{i i}+\rho \sum_{j} \beta_{i j}\right]
$$

Next, the first-order condition with respect to $\beta_{i \hat{i}}$ yields

$$
0=k \frac{\partial}{\partial \beta_{i \hat{i}}} C\left(\beta_{i}\right)=k \lambda \sigma_{\epsilon}^{2}\left[(1-\rho) \beta_{i \hat{i}}+\rho \sum_{j} \beta_{i j}\right],
$$


or equivalently,

$$
\beta_{i \hat{i}}=-\frac{\rho}{1-\rho} \sum_{j} \beta_{i j}
$$

Finally, the first-order condition with respect to $y_{i j}$ implies

$$
0=k \frac{\partial}{\partial \beta_{i j}} C\left(\beta_{i}\right) \frac{\partial \beta_{i j}}{\partial y_{i j}}+\gamma_{I} \frac{\partial \beta_{i j}}{\partial y_{j j}}=k \lambda \sigma_{\epsilon}^{2}\left[(1-\rho) \beta_{i j}+\rho \sum_{j} \beta_{i j}\right] \frac{1}{1-\delta}-\gamma_{I} \frac{\mu_{e} \delta}{1-\delta}
$$

which is equivalent to

$$
\beta_{i j}=-\frac{\rho}{1-\rho} \sum_{j} \beta_{i j}+\frac{\mu_{e} \delta \gamma_{I}}{k \lambda \sigma_{\epsilon}^{2}(1-\rho)}
$$

Summing (81) and (82),

$$
\sum_{j} \beta_{i j}=\beta_{i i}-\frac{n \rho}{1-\rho} \sum_{j} \beta_{i j}+\frac{\left(N-N_{I}\right) \mu_{e} \delta \gamma_{I}}{k \lambda \sigma_{\epsilon}^{2}(1-\rho)}
$$

and therefore, because $v=\frac{1}{n} \bar{\theta}\left(N-N_{I}\right) \mu_{e} \delta \gamma_{I}$,

$$
\begin{aligned}
& \beta_{i \hat{i}}=-\frac{\rho}{1-\rho} \sum_{j} \beta_{i j}=-\frac{1}{n} \bar{\theta}\left(\beta_{i i}+\frac{\left(N-N_{I}\right) \mu_{e} \delta \gamma_{I}}{k \lambda \sigma_{\epsilon}^{2}(1-\rho)}\right)=-\frac{1}{n} \bar{\theta} \beta_{i i}-\frac{v}{k \lambda \sigma_{\epsilon}^{2}(1-\rho)} \\
& \beta_{i j}=-\frac{1}{n} \bar{\theta} \beta_{i i}-\frac{v}{k \lambda \sigma_{\epsilon}^{2}(1-\rho)}+\frac{\mu_{e} \delta \gamma_{I}}{k \lambda \sigma_{\epsilon}^{2}(1-\rho)}=-\frac{1}{n} \bar{\theta} \beta_{i i}+\frac{v}{k \lambda \sigma_{\epsilon}^{2}(1-\rho)}\left(\frac{N-1}{\bar{\theta}\left(N-N_{I}\right)}-1\right)
\end{aligned}
$$

The final form of the results follow by noting that $k \lambda \sigma_{\epsilon}^{2}(1-\rho)=\frac{k \lambda \bar{\sigma}^{2}}{1+\frac{1}{n} \bar{\theta}}=\frac{1-y_{i i}^{*}}{\left(1+\frac{1}{n} \bar{\theta}\right) y_{i i}^{*}}$. Finally, from (80),

$$
\begin{aligned}
1+\alpha_{I} & =\beta_{i i}+k \lambda \sigma_{\epsilon}^{2}\left[(1-\rho) \beta_{i i}+\rho \sum_{j} \beta_{i j}\right] \\
& =\beta_{i i}+k \lambda \sigma_{\epsilon}^{2}(1-\rho)\left[\beta_{i i}+\frac{1}{n} \bar{\theta} \beta_{i i}+\frac{v}{k \lambda \sigma_{\epsilon}^{2}(1-\rho)}\right] \\
& =\left(1+k \lambda \bar{\sigma}^{2}\right) \beta_{i i}+v
\end{aligned}
$$


Note that without public disclosure, $\frac{\partial \beta_{j j}}{\partial y_{i j}}=0$, so that the last term in (82) is zero. Hence the solution is the same as with $v=0$, which matches the result of LEMMA 2. Note also, in equilibrium, the sensitivities for each agent should be consistent with the contract parameters; that is $\beta=\Delta y$ :

$$
\begin{aligned}
& (1-\delta) \beta_{i i}=y_{i i}-\delta \sum_{k} \mu_{i k} y_{k i}=y_{i i}-\delta\left(\mu_{I} y_{t}+\left(N-N_{I}\right) \mu_{e} y_{e}\right), \\
& (1-\delta) \beta_{i \hat{i}}=y_{i \hat{i}}-\delta \sum_{k} \mu_{i k} y_{k \hat{i}}=y_{t}-\delta\left(\mu_{t} y_{i i}+\left(\mu_{I}-\mu_{t}\right) y_{t}+\left(N-N_{I}\right) \mu_{e} y_{e}\right) \\
& (1-\delta) \beta_{i j}=y_{i j}-\delta \sum_{k} \mu_{i k} y_{k j}=y_{e}-\delta\left(\mu_{e} y_{i i}+\left(N_{I}-1\right) \mu_{e} y_{t}+\left(N-2 N_{I}\right) \mu_{e} y_{e}+\mu_{I} y_{e}\right)
\end{aligned}
$$

We thus have six linear equations we can solve to determine the six unknowns $\left(\beta^{D}, y^{D}\right)$. 


\section{References}

Aggarwal, R. K., and A. A. Samwick. 1999a. The Other Side of the Trade-Off: The Impact of Risk on Executive Compensation. Journal of Political Economy 107:65-105.

- 1999b. Executive Compensation, Strategic Competition, and Relative Performance Evaluation: Theory and Evidence. Journal of Finance 54:1999-2043.

Antle, R., and A. Smith. 1986. An Empirical Investigation of the Relative Performance Evaluation of Corporate Executives. Journal of Accounting Research 24:1-39.

Anton, M., F. Ederer, M. Gine, and M. Schmalz 2016. Common Ownership, Competition, and Top Management Incentives. Working paper, University of Michigan.

Barro, J., and R. Barro. 1990. Pay, Performance, and Turnover of Bank CEO's. Journal of Labor Economics 8: 448-81.

Bartling, B. 2011. Relative Performance or Team Evaluation? Optimal Contracts for OtherRegarding Agents. Journal of Economic Behavior \& Organization 79 183- 193.

Bartling, B., and F. von Siemens. 2010. The intensity of incentives in firms and markets: moral hazard with envious agents. Labour Economics 17 (2), 598-607.

Bellet, C. 2017. The Paradox of the Joneses: Superstar Houses and Mortgage Frenzy in Suburban America. London School of Economics, CEP Discussion Paper No 1462.

Bertrand M, Mullainathan S. 2001. Are CEOs rewarded for luck? The ones without principals are. Quarterly Journal of Economics 116(3):901-32.

Bouwman, C.H.S. 2013 The Geography of Executive Compensation. Wharton Financial Institutions Center working paper.

Card, D., A. Mas, E. Moretti, and E. Saez. 2012. Inequality at Work: The Effect of Peer Salaries on Job Satisfaction. American Economic Review: 2981-3003.

Carlsson,. F., G. Gupta and O. Johansson-Stenman. 2009. Keeping up with the Vaishyas? Caste and relative standing in India. Oxford Economic Papers 61: 52-73. 
Clark, A.E. 1996. L'utilité est-elle relative? Analyse à l'aide de données sur les ménages. Economie et Prévision, vol.121, pp. 151-164.

Clark, A.E. and A.J. Oswald. 1996. Satisfaction and comparison income. Journal of Public Economics 61: 359-81.

DeMarzo, P., M. Fishman, Z. He, and N. Wang. 2012. Dynamic Agency and the $q$ Theory of Investment. Journal of Finance 67 (6), 2295-2340.

Dohmen, T., A. Falk, K. Fliessbach, U. Sunde and B. Weber. 2011. Relative versus absolute income, joy of winning, and gender: Brain imaging evidence. Journal of Public Economics 95: 279-285.

Duchin, R., A. Goldberg, and D. Sosyura. 2016. Spillovers inside Conglomerates: Incentives and Capital. Review of Financial Studies, forthcoming.

Dur, R., and A. Glazer. 2008. Optimal contracts when a worker envies his boss. Journal of Law, Economics, and Organization 24 (1), 120-137.

Englmaier, F., and A. Wambach. 2010. Optimal Incentive Contracts under Inequity Aversion. Games and Economic Behavior 69 (2), 312-28.

Ederer, F. and A. Patacconi. 2010. Interpersonal Comparison, Status and Ambition in Organizations. Journal of Economic Behavior \& Organization 75 (2010) 348-363.

Ferrer-i-Carbonell, A. . 2005. Income and well-being: an empirical analysis of the comparison income effect. Journal of Public Economics 89: 997 - 1019.

Fershtman, C., H.K. Hvide, and Y. Weiss. 2003. A behavioral explanation of the relative performance evaluation puzzle. Annales d'Economie et de Statistique (71-72), 349-361.

Fliessbach, K., B. Weber, P. Trautner, T. Dohmen, U. Sunde, C. E. Elger and A. Falk. 2007. Social

Comparison Affects Reward-Related Brain Activity in the Human Ventral Striatum. Science 318 (5854): 1305-1308.

Frydman, C. and D. Jenter. 2010. CEO Compensation. Annual Review of Financial Economics 2: $75-102$. 
Garcia, D. and G. Strobl 2011. Relative Wealth Concerns and Complementarities in Information Acquisition. Review of Financial Studies 24:169-207.

Gartenberg, C. and J. Wulf. 2016. Pay Harmony? Peer Comparison and Performance Compensation in Multi-business Firms. Working paper, Harvard Business School.

Garvey, G., and T. Milbourn. 2003. Incentive Compensation When Executives Can Hedge the Market: Evidence of Relative Performance Evaluation in the Cross Section. Journal of Finance 58:1557-81.

Garvey, G., and T. Milbourn. 2006. Asymmetric Benchmarking in Compensation: Executives Are Rewarded for Good Luck But Not Penalized for Bad. Journal of Financial Economics 82:197-225.

Ghazala, A. and N. Iriberri. 2010. The importance of relative performance feedback information: Evidence from a natural experiment using high school students. Journal of Public

Economics 94: 435-452.Gibbons, R., and K.J. Murphy. 1990. Relative Performance Evaluation for Chief Executive Officers. Industrial and Labor Relations Review 43(3): 30S-51S.

Gipper, B., 2016, Assessing the Effects of Disclosing Management Compensation, Working paper Stanford University.

Goel, A.M., and A.V. Thakor. 2005. Green with Envy: Implications for Corporate Investment Distortions. Journal of Business 78(6), 2005, pp. 2255-2288.

Goel, A.M., and A.V. Thakor. 2010. Do envious CEOs cause merger waves? Review of Financial Studies 23, 487-517.

Gopalan, R., T. Milbourn and F. Song. 2010. Strategic Flexibility and the Optimality of Pay for Sector Performance. Review of Financial Studies 23: 2060-2098.

Goukasian, L. and X. Wan. 2010. Optimal Incentive Contracts Under Relative Income Concerns. Mathematics and Financial Economics 4:57-86.

Hall, B.J., and J.B. Liebman. 1998. Are CEOs Really Paid Like Bureaucrats? Quarterly Journal of Economics 3: 653-691. 
Heffetz, O. and R.H. Frank. 2011. Preferences for Status: Evidence and Economic Implications. In Jess Benhabib, Alberto Bisin, and Matthew Jackson (eds.), Handbook of Social Economics (vol. 1A, pp. 69-91). Amsterdam: Elsevier.

Himmelberg, C.P., and R.G. Hubbard. 2000. Incentive pay and the market for CEOs: An analysis of pay-for-performance sensitivity. Working paper Columbia University.

Ibert, M., R. Kaniel, S. Van Nieuwerburgh, and R. Vestman. 2018. Are Mutual Fund Managers Paid For Investment Skill? Review of Financial Studies 31(2): 715-772.

Janakiraman, S. N., R. A. Lambert, and D. F. Larcker. 1992. An Empirical Investigation of the Relative Performance Evaluation Hypothesis. Journal of Accounting Research 30:53-69.

Jenter, D. and F. Kanaan. 2014. CEO Turnover and Relative Performance Evaluation. Journal of Finance, forthcoming.

Jensen, M. C., and K. J. Murphy. 1990. Performance Pay and Top-Management Incentives. Journal of Political Economy 98:225-64.

Joh, S.W. 1999. Strategic Managerial Incentive Compensation in Japan: Relative Performance Evaluation and Product Market Collusion. The Review of Economics and Statistics 81(2):303-313.

Knight, J., L. Song and R. Gunatilaka. 2009. Subjective well-being and its determinants in rural China. China Economic Review 20: 635-649.

Koszegi, B. 2014. Behavioral Contract Theory. Journal of Economic Literature 52(4):10751118.

Liu, Qi and Bo Sun. 2016. Relative Wealth Concerns, Executive Compensation, and Systemic Risk-Taking. Working paper, Federal Reserve Board of Governors.

Luttmer, E. 2005. Neighbors as negatives: Relative earnings and well-being. Quarterly Journal of Economics 120: 963-1002.

Mas, A., 2016, Does Disclosure affect CEO Pay Setting? Evidence from the Passage of the 1934 Securities and Exchange Act, Working paper Princeton University. 
Miglietta, S. 2008. Incentive Contracts and Status-Concerned Agents. Working Paper BI Norwegian School of Management.

Neumark, D., and A. Postlewaite.1998. Relative income concerns and the rise in married women's employment. Journal of Public Economics 70: 157-183.

Oyer, P. 2004. Why Do Firms Use Incentives That Have No Incentive Effects? Journal of Finance 59, 1619-1640.

Park, Y.W., T. Nelson, and M.R. Huson, 2001, Executive Pay and Disclosure Environment: Canadian Evidence, Journal of Financial Research, 347-365.

Perry, T., and M., Zenner, 2002, Pay for performance? Government regulation and the structure of compensation contracts, Journal of Financial Economics, 435-488.

Schmidt, C., 2012, Does Transparency Increase Executive Compensation?, Working paper, SSRN.

Shue, K. 2013. Executive Networks and Firm Policies: Evidence from the Random Assignment of MBA Peers, Review of Financial Studies 26, 1401-1442.

Silva, R. 2013. Internal Labor Markets and Investment in Conglomerates. Working Paper, London Business School. 